DOI 10.4171/JEMS/325

Jaume Amorós · Mònica Manjarín · Marcel Nicolau

\title{
Deformations of Kähler manifolds with nonvanishing holomorphic vector fields
}

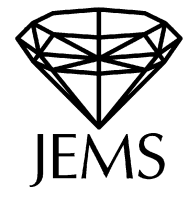

\section{To Marco Brunella, in memoriam}

Received February 26, 2010 and in revised form July 9, 2010

\begin{abstract}
We study compact Kähler manifolds $X$ admitting nonvanishing holomorphic vector fields, extending the classical birational classification of projective varieties with tangent vector fields to a classification modulo deformation in the Kähler case, and biholomorphic in the projective case. We introduce and analyze a new class of tangential deformations, and show that they form a smooth subspace in the Kuranishi space of deformations of the complex structure of $X$. We extend Calabi's theorem on the structure of compact Kähler manifolds $X$ with $c_{1}(X)=0$ to compact Kähler manifolds with nonvanishing tangent fields, proving that any such manifold $X$ admits an arbitrarily small tangential deformation which is a suspension over a torus, that is, a quotient of $F \times \mathbb{C}^{S}$ fibering over a torus $T=\mathbb{C}^{S} / \Lambda$. We further show that either $X$ is uniruled or, up to a finite Abelian covering, it is a small deformation of a product $F \times T$ where $F$ is a Kähler manifold without tangent vector fields and $T$ is a torus. A complete classification when $X$ is a projective manifold, in which case the deformations may be omitted, or when $\operatorname{dim} X \leq s+2$, is also given. As an application, it is shown that the study of the dynamics of holomorphic tangent fields on compact Kähler manifolds reduces to the case of rational varieties.
\end{abstract}

Keywords. Kähler manifold, deformation, vector field, Fujiki manifold

\section{Contents}

1. Groups of automorphisms of Kähler manifolds . . . . . . . . . . . . . . . . . 1001

2. Locally free actions on Kähler and Fujiki manifolds . . . . . . . . . . . . . . . . 1005

3. Projective manifolds with nonvanishing tangent fields . . . . . . . . . . . . . . . 1008

4. Tangential deformations of locally free holomorphic actions . . . . . . . . . . . 1011

5. Tangential deformations of locally free $\mathbb{C}^{S}$-actions on Kähler and Fujiki manifolds . . . 1013

6. The approximation theorem . . . . . . . . . . . . . . . . . . . 1021

7. Structure of Kähler manifolds with nonvanishing vector fields . . . . . . . . . . . . . 1025

8. Locally free $\mathbb{C}^{S}$-actions on Kähler manifolds with small codimension . . . . . . . . . . 1033

9. Dynamics of holomorphic vector fields . . . . . . . . . . . . . . . . 1036

References . . . . . . . . . . . . . . . . . . . . . . . . . 1039

J. Amorós: Departament de Matemàtica Aplicada I, Universitat Politècnica de Catalunya,

Diagonal 647, E-08028 Barcelona, Spain; e-mail: jaume.amoros@upc.edu

M. Manjarín, M. Nicolau: Departament de Matemàtiques, Universitat Autònoma de Barcelona, 08193 Bellaterra, Spain; e-mail: monica@mat.uab.cat, nicolau@mat.uab.cat

Mathematics Subject Classification (2010): Primary 32G07, 32M; Secondary 14M17, 14L27, 32J27, 37F75 


\section{Introduction}

This article is devoted to the study of compact Kähler manifolds admitting nonvanishing holomorphic vector fields or, what is equivalent, endowed with a locally free holomorphic $\mathbb{C}^{S}$-action for a certain $s>0$. We introduce a particular type of deformations of such manifolds and, as an application, we describe quite precisely their structure. Our study relies on the work carried out simultaneously and independently by A. Fujiki [16] and D. Lieberman [28]. We use especially their deep result on the structure of the group of holomorphic automorphisms of a compact Kähler manifold (cf. Theorem 1.2).

Our work is a continuation of the classical study of complex projective manifolds with tangent vector fields, which led to their birational classification up to finite covering, successively developed by F. Severi, R. Hall and D. Lieberman ([31], [20], [27]). The conclusions were summarized in Theorem 1 of [27] as

Theorem 0.1. Let $X$ be a complex projective manifold with a holomorphic tangent vector field $v$. Then:

(a) $X$ has a finite étale Abelian covering $X^{\prime}$ which is birational to a product $M \times T \times$ $\mathbb{C} P^{r}$, where $M$ is another projective manifold, $T$ an Abelian variety, and $\mathbb{C} P^{r}$ the projective space of dimension $r \geq 0$, such that the lift of the tangent vector field $v$ to $X^{\prime}$ has trivial component in $T M$.

(b) If the tangent vector field $v$ on $X$ vanishes at some point then $X$ is ruled.

In Theorem 3.2 below we continue this classification in the case of nonvanishing vector fields, obtaining a classification up to biholomorphism by using suspensions over Abelian varieties (see Example 2.4) and repeated étale Abelian coverings in order to exhaust all tangent vector fields.

Tangent vector fields play a role in the classification of algebraic varieties, specially in the case of Kodaira dimension 0. The earliest indication of this fact known to the authors is the result stated by Eugenio Calabi in [8]. In that article, E. Calabi formulated his celebrated conjecture about Kähler manifolds admitting Ricci-flat metrics and, under the assumption that the conjecture was true, he proved the result stated as Theorem 0.2 below. This theorem describes the structure of compact Kähler manifolds with trivial first Chern class, i.e. with $c_{1}(X)=0$. It was proved for projective manifolds by Y. Matsushima in [30] using previous results by A. Lichnerowicz, but without the assumption of Calabi's conjecture, and extended to the general Kähler case by F. A. Bogomolov [6] and independently by Lieberman [28].

Theorem 0.2 (Calabi, [8], [30], [6], [28]). Let X be a compact Kähler manifold with $c_{1}(X)=0$ and $b_{1}(X)>0$. Then $X$ admits, as a finite étale covering, a product $X^{\prime}=$ $F \times T$, with $T$ a complex torus of real dimension $b_{1}\left(X^{\prime}\right)$ and $F$ a Kähler manifold with $c_{1}(F)=0$ and $b_{1}(F)=0$. Moreover, $X^{\prime}$ is a regular covering space of $X$ and the group of covering transformations is solvable.

This theorem was improved by Beauville [2], who completely describes the topology and the geometry of the fiber $F$. 
The proof of Calabi's theorem may be succinctly described as follows: Use the triviality of the canonical bundle to show that $X$ (or a finite covering of it) has $q=\frac{1}{2} b_{1}(X)$ linearly independent nonvanishing tangent vector fields; check that the Albanese morphism is onto, the tangent vector fields are lifts from the tangent bundle on the Albanese torus, and they make $X$ (or a finite covering of it) a suspension with trivial monodromy over the Albanese torus.

In this work we develop a refinement of the above argument to prove an extension to general Kähler manifolds of Calabi's theorem, namely our Theorem 3.2 and Proposition 7.3 may be condensed as:

Theorem 0.3. Let $X$ be a compact Kähler manifold with s non vanishing tangent vector fields $v_{1}, \ldots, v_{s}$, defining a locally free $\mathbb{C}^{s}$-action. Then $X$ admits a small deformation $X_{\epsilon}$, which is a suspension over an $s$-dimensional torus $T$.

Furthermore, there is a finite Abelian covering $X_{\epsilon}^{\prime}$ of a small deformation $X_{\epsilon}$ of $X$, a torus $T^{\prime}$ of dimension $s^{\prime} \geq s$ and a compact Kähler manifold $F$ without nonvanishing vector fields in such a way that:

(i) if $\operatorname{kod}(X) \geq 0$ then $X_{\epsilon}^{\prime}=F \times T^{\prime}$ and $F$ has no vector fields,

(ii) if $\operatorname{kod}(X)=-\infty$ then $X_{\epsilon}^{\prime}$ is a topologically trivial suspension over $T^{\prime}$ and fiber $F$.

In both cases $\operatorname{kod}(F)=\operatorname{kod}(X)$. If $X$ is a complex projective manifold one may omit the small deformation of the complex structure, and the above decomposition holds for $X_{\epsilon}=X$.

Here, $\operatorname{kod}(X)$ denotes the Kodaira dimension of $X$. The definition of a suspension manifold is recalled in Example 2.4.

We notice that a locally free holomorphic $\mathbb{C}^{s}$-action, i.e. a holomorphic $\mathbb{C}^{s}$-action for which every isotropy group is discrete, is determined by $s$ holomorphic vector fields $v_{1}, \ldots, v_{s}$ generating an $s$-dimensional Abelian Lie algebra a such that each vector field $v$ in $\mathfrak{a}$ different from zero is a nonvanishing vector field. On a compact Kähler manifold $X$, the set $\mathfrak{h}^{1}$ of vector fields having zeros is an ideal of the Lie algebra $\mathfrak{h}$ of holomorphic vector fields on $X$, there is a direct sum decomposition $\mathfrak{h}=\mathfrak{h}^{1} \oplus \mathfrak{a}$, where $\mathfrak{a}$ is an Abelian subalgebra of $\mathfrak{h}$, and $X$ admits a locally free holomorphic $\mathbb{C}^{s}$-action if and only if $\operatorname{dim} \mathfrak{a}>0$ (cf. Propositions 1.4 and 1.6). Hence the hypothesis in the above theorem is fulfilled whenever $s=\operatorname{dim}\left(\mathfrak{h} / \mathfrak{h}^{1}\right)>0$. This is the case, in particular, if $X$ admits a nonvanishing vector field.

The small deformation of the complex structure of $X$ cannot be avoided in the general Kähler case if $c_{1}(X) \neq 0$, as shown by the examples discussed in Section 7.

On the other hand, for $X$ compact Kähler with $c_{1}(X)=0$, the dimension of the space of holomorphic vector fields on $X$ is $b_{1}(X) / 2$ and this fact also renders unnecessary the small deformation of complex structure, thus Corollary 6.8 to our Theorem 0.3 refines Calabi's theorem, as we show that the group of covering transformations is in fact Abelian. This refinement was established in [6] in the case when $X$ is a complex projective manifold.

Besides this extension of Calabi's theorem from Calabi-Yau manifolds to any Kähler manifolds with nonvanishing tangent vector fields, the authors hope that the results of 
this work may have further applications in the case of manifolds of Kodaira dimension zero. K. Ueno [32, §11] made a conjecture which is the extension of Calabi's one to this setting, and was refined by János Kollár [24, 4.16] as:

Conjecture 0.4 (Ueno, Kollár). Let $X$ be a smooth, projective variety with $\operatorname{kod}(X)=0$. Then $X$ has a finite, étale covering $\tilde{X}$ such that $\tilde{X}$ is birational to the product of an Abelian variety and a simply connected variety $F$ with $\operatorname{kod}(F)=0$.

Corollary 8.10 is a modest contribution to this problem, establishing the conjecture if one has $\operatorname{dim} X-2$ linearly independent nonvanishing tangent vector fields, a particular case being threefolds with nonvanishing vector fields.

This corollary is a particular case of the classification of compact Kähler and projective manifolds with $\operatorname{dim} X-2$ linearly independent nonvanishing tangent vector fields carried out in Section 8 based on Theorem 0.3 and the classification of curves and surfaces.

In Section 9 we extend the results in [28] concerning the dynamical properties of a holomorphic tangent vector field $v$ on a compact Kähler manifold $X$. Applying Theorem 0.3 , we show that the dynamical system $(X, v)$ becomes trivial after taking a finite unramified covering space of $X$ if $\operatorname{kod}(X) \geq 0$ and that, in all cases, the dynamics of the system reduce to the dynamics of an Abelian group $\mathbb{C}^{p} \times\left(\mathbb{C}^{*}\right)^{q}$ acting on a rational variety (Theorem 9.2).

In order to achieve all the results described above, we introduce the notion of tangential deformation of a holomorphic group action. A locally free holomorphic action of a (connected) complex Lie group $G$ on a complex manifold $X$ defines a holomorphic foliation $\mathcal{F}$ on $X$ whose leaves are the orbits of the action. $G$-equivariant deformations of $X$ change the complex structure of $X$ as well as the holomorphic foliation $\mathcal{F}$. The existence of a versal space of deformations for equivariant deformations of compact complex manifolds endowed with a holomorphic action was proved by Cathelineau [13]. In this article we consider equivariant deformations of the locally free $G$-action for which the foliation $\mathcal{F}$ keeps fixed its holomorphic transverse structure. This type of deformations, called tangential deformations of the action, also admit a versal space of deformations whose tangent space at the origin is naturally identified to the space of infinitesimal tangential deformations (Theorem 4.3).

We focus on the case where $X$ is a Kähler manifold. Under that hypothesis and as a consequence of the above mentioned theorem by Fujiki and Lieberman, the group $G$ is necessarily Abelian. For a given locally free holomorphic $\mathbb{C}^{s}$-action on a compact Kähler manifold $X$ we construct a family $X_{R}$ of tangential deformations of the action parametrized by a smooth space $R$ with the following properties:

(1) $R$ is an open subset of a Euclidean space $\mathbb{C}^{N}$, complementary to an affine real algebraic variety.

(2) The family $X_{R}$ is versal at each point $r \in R$ (Theorem 5.5 and Proposition 5.19).

(3) Each element $X_{r}$ with $r \in R$ is a Kähler manifold (Theorem 5.17).

We emphasize that $R$ is a (real) Zariski open set and not merely a small open ball of $\mathbb{C}^{N}$. 
Looking at general properties of tangential deformations of the action, we prove that the Kodaira dimension $\operatorname{kod}(X)$ of $X$ is constant under tangential deformations. We also relate $R$ to the Kuranishi space $\mathcal{K}_{X}$ of $X$ by showing that the forgetful map

$$
R \rightarrow \mathcal{K}_{X}
$$

has a smooth image of dimension $s \cdot b_{1}(X) / 2$. In particular this shows the following (cf. Theorem 5.6):

Theorem 0.5. The space $H^{1}\left(X, \Theta_{X}\right)$ of infinitesimal deformations of a compact Kähler manifold $X$ endowed with a locally free $\mathbb{C}^{S}$-action contains a subspace of dimension $\ell=s \cdot b_{1}(X) / 2$ of unobstructed infinitesimal deformations. Consequently, the Kuranishi space $\mathcal{K}_{X}$ contains a smooth subspace of dimension $\ell$.

Many of the results of this article are also valid for manifolds $X$ belonging to the class $\mathcal{C}$, introduced by A. Fujiki and whose elements are those manifolds that are bimeromorphic to Kähler manifolds. Hence some of the results are stated in that context. However, we do not dispose of a characterization of those suspension manifolds that are in $\mathcal{C}$ (cf. Remark 2.5). Moreover, $\mathcal{C}$ is not stable under small deformations and for these reasons we do not know if the structure theorems of Section 7 are valid for Fujiki manifolds and we are able to prove them only in the case of Kähler manifolds.

Even if not explicitly stated, all the group actions considered along this article are holomorphic and the covering spaces are always unramified (étale).

\section{Groups of automorphisms of Kähler manifolds}

Let $X$ be a compact complex manifold of dimension $n$. All along this article, with the only exception of Section 4, the manifold $X$ will be assumed to be Kähler or, more generally, to belong to the class $\mathcal{C}$ introduced by A. Fujiki in [15]. We recall that a manifold belongs to the class $\mathcal{C}$ (or the Fujiki class) if it is bimeromorphic to a Kähler manifold. Notice that Moishezon manifolds are in $\mathcal{C}$ and that a submanifold of a Fujiki manifold is again in $\mathcal{C}$. Recently, Demailly and Paun have characterized compact manifolds in $\mathcal{C}$ as those manifolds carrying a Kähler current (cf. [14]).

Compact manifolds $X$ in the class $\mathcal{C}$ retain many of the properties of compact Kähler manifolds. In this article we will make use of the following ones: (i) holomorphic forms on $X$ are closed, (ii) there are $\mathbb{C}$-antilinear isomorphisms $H^{q}\left(X, \Omega_{X}^{p}\right) \equiv H^{p}\left(X, \Omega_{X}^{q}\right)$, (iii) the Hodge theorem holds, i.e. $H^{m}(X, \mathbb{C}) \equiv \bigoplus_{p+q=m} H^{q}\left(X, \Omega_{X}^{p}\right)$ and (iv) $H^{1}(X, \mathbb{C})$ is isomorphic to $H^{1}(\operatorname{Alb}(X), \mathbb{C})$, where $\operatorname{Alb}(X)$ is the Albanese torus of $X$. For the proof of the above statements and a general account on the properties of Fujiki manifolds we refer to [16] and [17]. In sharp contrast, the class $\mathcal{C}$ is not stable under small deformations (cf. [9] and [25]).

Let $\mathfrak{h}_{X}$ be the Lie algebra of holomorphic vector fields on $X$ and $\mathfrak{h}_{X}^{1}$ the subspace of vector fields annihilated by all the holomorphic 1-forms on $X$. Since global holomorphic 1 -forms are closed, $\mathfrak{h}_{X}^{1}$ is an ideal of $\mathfrak{h}_{X}$ containing the derived subalgebra $\left[\mathfrak{h}_{X}, \mathfrak{h}_{X}\right]$ of $\mathfrak{h}_{X}$. 
The following characterization of $\mathfrak{h}_{X}^{1}$ obtained by Carrell and Lieberman [11] was extended by Fujiki [15] to the class $\mathcal{C}$.

Theorem 1.1 (Carrell-Lieberman [11]). Let X be a compact Kähler manifold. Then $\mathfrak{h}_{X}^{1}$ is the vector space of holomorphic vector fields with zeros. In particular, if $X$ admits $a$ nonvanishing holomorphic vector field $v$, then there is a holomorphic 1-form $\alpha$ on $X$ such that $\alpha(v)=1$.

Let $\phi: X \rightarrow \operatorname{Alb}(X)$ be the natural map from $X$ to its $\operatorname{Albanese}$ torus $\operatorname{Alb}(X)$. Each element of the group $\operatorname{Aut}_{\mathbb{C}}(X)$ of holomorphic automorphisms of $X$ induces an automorphism of $\mathrm{Alb}(X)$ and the correspondence

$$
\Phi: \operatorname{Aut}_{\mathbb{C}}(X) \rightarrow \operatorname{Aut}_{\mathbb{C}}(\operatorname{Alb}(X))
$$

is a group morphism. The map $\phi$ is equivariant with respect to the action of $\operatorname{Aut}_{\mathbb{C}}(X)$ on $\operatorname{Alb}(X)$ induced by $\Phi$. The connected component of the identity $\operatorname{Aut}_{\mathbb{C}}^{0}(X)$ of $\operatorname{Aut}_{\mathbb{C}}(X)$ is mapped by $\Phi$ into $\operatorname{Aut}_{\mathbb{C}}^{0}(\operatorname{Alb}(X)) \cong \operatorname{Alb}(X)$ and its image is a subtorus $T_{X}$ of $\operatorname{Alb}(X)$. Notice that $T_{X}$ is contained in the image $\phi(X)$ of $X$ into $\operatorname{Alb}(X)$.

The following result was obtained independently and at the same time by A. Fujiki and by D. Lieberman. The latter proved it for Kähler manifolds and the former for manifolds in the class $\mathcal{C}$.

Theorem 1.2 (Fujiki [16], Lieberman [28]). Let X be a compact complex manifold that is Kähler or, more generally, that belongs to the Fujiki class. Then there is an exact sequence of groups

$$
1 \rightarrow L \rightarrow \operatorname{Aut}_{\mathbb{C}}^{0}(X) \stackrel{\Phi}{\rightarrow} T_{X} \rightarrow 1
$$

where $L$ is a linear algebraic group (so with finitely many connected components) with Lie algebra $\mathfrak{h}_{X}^{1}$. Moreover, there is a group morphism $\iota: \mathbb{C}^{r} \rightarrow \operatorname{Aut}_{\mathbb{C}}^{0}(X)$ such that the composition $\Phi \circ \iota$ is the universal covering of $T_{X}$.

Corollary 1.3. If $\mathfrak{h}_{X}^{1}=0$ then $\operatorname{Aut}_{\mathbb{C}}^{0}(X)$ is a complex torus.

Notice that Theorem 1.1 is also a corollary of Theorem 1.2. Another consequence of the theorem is the following:

Proposition 1.4. There is an Abelian Lie subalgebra $\mathfrak{a}$ of $\mathfrak{h}_{X}$ such that

$$
\mathfrak{h}_{X}=\mathfrak{h}_{X}^{1} \oplus \mathfrak{a},
$$

where the direct sum is in the sense of vector spaces.

Remarks 1.5. (a) We denote $s=\operatorname{dim}_{\mathbb{C}} T_{X}=\operatorname{dim}_{\mathbb{C}} \mathfrak{a}=\operatorname{dim}_{\mathbb{C}} \mathfrak{h}_{X} / \mathfrak{h}_{X}^{1}$. Then

$$
s \leq \operatorname{dim}_{\mathbb{C}} \operatorname{Alb}(X)=b_{1}(X) / 2 \quad \text { and } \quad s \leq \operatorname{dim}_{\mathbb{C}}(X) .
$$

(b) The subalgebra $\mathfrak{a}$ is not unique. Such a Lie subalgebra can be characterized as a vector subspace of $\mathfrak{h}_{X}$ of maximal dimension generated by nonvanishing commuting vector fields. 
(c) Let $v_{1}, \ldots, v_{s}$ be a basis of $\mathfrak{a}$. Then the vector fields $v_{1}, \ldots, v_{s}$ are linearly independent at each point of $X$.

(d) The choice of a basis $v_{1}, \ldots, v_{s}$ of $\mathfrak{a}$ determines an action of $\mathbb{C}^{s}$ on $X$ which is locally free, i.e. an action which is injective at the Lie algebra level or, what is equivalent, which has discrete isotropy groups. Conversely, given such a locally free action $\varpi$ : $\mathbb{C}^{m} \times X \rightarrow X$, of maximal rank (i.e. $m$ is maximal), one has $m=s$ and the fundamental vector fields of the action generate a subalgebra of $\mathfrak{h}_{X}$ complementary to $\mathfrak{h}_{X}^{1}$. If the orbits are given by a torus action, i.e. the action factorizes through a torus $T=\mathbb{C}^{s} / \Lambda$, then $\mathfrak{a}$ is contained in the center of $\mathfrak{h}_{X}$.

The following result is stated without proof in [28]. The proof we give here is due to F. Touzet.

Proposition 1.6. The Abelian subalgebra a of $\mathfrak{h}_{X}$ satisfying (2) can be chosen in the center of $\mathfrak{h}_{X}$.

Proof. First we notice that $L$ can be assumed to be connected just by replacing $T_{X}$ by a finite covering, which is again a torus. Let $\operatorname{Aut}^{0}\left(\mathfrak{h}_{X}^{1}\right)$ be the identity component of the automorphism group of the complex Lie algebra $\mathfrak{h}_{X}^{1}=\operatorname{Lie}(L)$. Since the group $L$ is normal in $G=\operatorname{Aut}_{\mathbb{C}}^{0}(X)$, the adjoint representation

$$
\psi: G \rightarrow \operatorname{Aut}^{0}\left(\mathfrak{h}_{X}^{1}\right), \quad g \mapsto \operatorname{Ad}_{g},
$$

is well defined and induces a group morphism

$$
\tilde{\psi}: T_{X} \rightarrow H=\operatorname{Aut}^{0}\left(\mathfrak{h}_{X}^{1}\right) / \operatorname{Ad}(L)
$$

Notice that $\operatorname{Ad}(L)$ is closed in $\operatorname{Aut}^{0}\left(\mathfrak{h}_{X}^{1}\right)$, as $L$ is an algebraic group, and $\operatorname{Ad}(L)$ is a normal subgroup of $\operatorname{Aut}^{0}\left(\mathfrak{h}_{X}^{1}\right)$, as $\operatorname{Lie}(\operatorname{Ad}(L))$ is an ideal of $\operatorname{Der}^{0}\left(\mathfrak{h}_{X}^{1}\right)$. Therefore the connected group $\operatorname{Ad}(L)$ is a normal and closed subgroup of the algebraic group $\operatorname{Aut}^{0}\left(\mathfrak{h}_{X}^{1}\right)$. Hence the quotient $H$ is also a linear algebraic group (cf. [22]) and the image under $\psi$ of the compact group $T_{X}$ is necessarily constant, thus equal to the identity. As a consequence, $\psi$ has image contained in $\operatorname{Ad}(L)$. We deduce that, for each $v \in \mathfrak{h}_{X}$, there is $v_{1}=v_{1}(v) \in \mathfrak{h}_{X}^{1}$ such that

$$
[v, w]=\left[v_{1}, w\right] \quad \forall w \in \mathfrak{h}_{X}^{1},
$$

that is, $v-v_{1}$ commutes with all the elements in $\mathfrak{h}_{X}^{1}$.

We now consider $v \in \mathfrak{h}_{X}$ with the property that $\Phi(\{\exp t v\})$ is dense in the torus $T_{X}$. Then $v-v_{1}$ has the same property. Hence the closure $A_{v-v_{1}}$ of the group $\left\{\exp t\left(v-v_{1}\right)\right\}$ is an Abelian subgroup of $G$ that is mapped onto $T_{X}$ by $\Phi$. This implies that $v-v_{1}$ is in the center of $\mathfrak{h}_{X}$ and therefore $A_{v-v_{1}}$ is in the center of $G$. Now we take a group morphism $\iota: \mathbb{C}^{r} \rightarrow A_{v-v_{1}}$ with the property that the composition $\Phi \circ \iota$ is the universal covering of $T_{X}$. Then the morphism $\iota$ determines an Abelian subalgebra $\mathfrak{a}$ of $\mathfrak{h}_{X}$ with the required properties. 
Remarks 1.7. (a) It follows from the above proposition that each nonvanishing holomorphic vector field $v$ on $X$ is included in an Abelian Lie algebra a satisfying (2).

(b) Assume that the subalgebra $\mathfrak{a}$ of $\mathfrak{h}$ with the properties of Proposition 1.4 has been fixed and set $\mathfrak{a}=\left\langle v_{1}, \ldots, v_{s}\right\rangle$. The space of holomorphic 1-forms on $X$ can be decomposed as

$$
H^{0}\left(X, \Omega_{X}^{1}\right)=\left\langle\alpha^{1}, \ldots, \alpha^{s}\right\rangle \oplus\left\langle\beta^{1}, \ldots, \beta^{p-s}\right\rangle,
$$

where $p=b_{1}(X) / 2=\operatorname{dim}_{\mathbb{C}} \operatorname{Alb}(X)$, and the forms $\alpha^{i}$ and $\beta^{k}$ satisfy $\alpha^{i}\left(v_{j}\right)=\delta_{j}^{i}$ and $\beta^{k}\left(v_{j}\right)=0$ for each $j=1, \ldots, s$.

Although the forms $\alpha^{i}$ are not uniquely determined unless $p=s$, the subspace $\left\langle\beta^{1}, \ldots, \beta^{p-s}\right\rangle$ is canonically associated to the complex manifold $X$. In particular, it does not depend on the choice of the subalgebra $\mathfrak{a}$. In fact, $\left\langle\beta^{1}, \ldots, \beta^{p-s}\right\rangle$ is just the kernel of the natural morphism

$$
H^{0}\left(X, \Omega_{X}^{1}\right) \rightarrow H^{0}\left(X, \Theta_{X}\right)^{\vee}
$$

where $\Theta_{X}$ denotes the sheaf of germs of holomorphic vector fields on $X$.

A manifold $X$ will be called ruled if it is bimeromorphic to a geometrically ruled manifold, that is if $X$ is bimeromorphic to the total space of a fiber bundle $Y \rightarrow B$, which is analytically locally trivial over a complex manifold $B$, has fiber a complex projective space $\mathbb{C} P^{k}$ and has changes of trivialization in the sheaf $\operatorname{PGL}\left(k+1, \mathcal{O}_{B}\right)$. For $X$ algebraic this is equivalent, by the GAGA theorem, to $X$ being bimeromorphic to the trivial bundle $\mathbb{C} P^{k} \times B$ but this equivalence is no longer true in the setting of Kähler manifolds (cf. [16]). A manifold $X$ will be called uniruled if every point $x \in X$ lies in a rational curve $C \subset X$.

Theorem 1.8 (Fujiki [16]). If a compact manifold $X$ in the class $\mathcal{C}$ admits a non-trivial holomorphic vector field $v$ with $v_{x}=0$ for some point $x \in X$, then $X$ is uniruled.

Remark 1.9. If the manifold $X$ in Theorem 1.8 is complex projective then it follows from the birational classification theorem (Theorem 0.1) that $X$ is ruled. In [28], Lieberman states that if $X$ is a compact Kähler manifold then it is also ruled. In the particular case when the automorphism group of $X$ includes a $\mathbb{C}^{*}$-action this was proved by Carrell and Sommese [12]. The authors have not been able to complete Lieberman's argument from [28] in the general compact Kähler case.

We end this section by recalling the following result of Lieberman concerning the Kodaira dimension, $\operatorname{kod}(X)$, of $X$ and the dimension of the Lie algebra $\mathfrak{h}_{X}$.

Theorem 1.10 (Lieberman [28]). Let X be a compact Kähler manifold. Then

$$
\operatorname{dim}_{\mathbb{C}} \mathfrak{h}_{X}+\operatorname{kod}(X) \leq \operatorname{dim}_{\mathbb{C}} X
$$




\section{Locally free actions on Kähler and Fujiki manifolds}

Let $X$ be a compact manifold endowed with a locally free $G$-action. In case $X$ is a Kähler or Fujiki manifold there are very few possibilities for the Lie group $G$. F. Bosio has noted the following corollary of the above mentioned theorem of Carrell and Lieberman.

Theorem 2.1 (Bosio [7]). Let $G$ be a connected complex Lie group acting locally freely on a compact manifold $X$ belonging to the class $\mathcal{C}$. Then $G$ is Abelian.

Proof. Theorem 1.1 implies that the induced action of $G$ on the Albanese torus $\operatorname{Alb}(X)$ is also locally free.

Here are some examples of compact complex manifolds naturally endowed with locally free $\mathbb{C}^{s}$-actions.

Example 2.2 (Tori). Complex tori, that is, $\mathbb{T}^{s}=\mathbb{C}^{s} / \Lambda$ where $\Lambda \cong \mathbb{Z}^{s}$ is a lattice, are the only compact manifolds $X$ with a locally free $\mathbb{C}^{s}$-action such that $s=\operatorname{dim}_{\mathbb{C}} X$.

Example 2.3 (Principal torus fibrations). The total space $X$ of a principal torus bundle

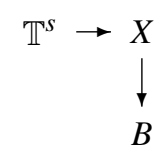

where $B$ is a compact complex manifold, is endowed with a natural action of $\mathbb{T}^{s}$ and therefore of $\mathbb{C}^{s}$. If $X$ is a Kähler manifold then the Kähler metric can be made invariant by averaging it with respect to the action by the compact group $\mathbb{T}^{s}$. Hence the base space $B$ is necessarily a Kähler manifold. Furthermore, it will be seen below (cf. Remark 2.7(a)) that the principal bundle is flat. Conversely, if $B$ is a Kähler manifold and the bundle is flat then the total space is a Kähler manifold. This follows from a theorem of Blanchard [5, Théorème principal II].

Example 2.4 (Suspensions over complex tori). Let $\rho: \Lambda \rightarrow \operatorname{Aut}_{\mathbb{C}}(F)$ be a group representation, where $\Lambda=\left\langle\tau_{1}, \ldots, \tau_{2 s}\right\rangle \cong \mathbb{Z}^{2 s}$ is a lattice of $\mathbb{C}^{s}$ and $F$ is a compact complex manifold, and denote $f_{i}=\rho\left(\tau_{i}\right) \in \operatorname{Aut}_{\mathbb{C}}(F)$. The suspension of the representation $\rho$ is the manifold $X=F \times{ }_{\Lambda} \mathbb{C}^{s}$ obtained as the quotient of the product $F \times \mathbb{C}^{s}$ by the equivalence relation defined by the diagonal action of $\Lambda$, that is,

$$
(x, z) \sim\left(f_{i}(x), z+\tau_{i}\right) \text { for } i=1, \ldots, 2 s .
$$

The suspension $X=F \times{ }_{\Lambda} \mathbb{C}^{s}$ fibers over the torus $\mathbb{T}^{s}=\mathbb{C}^{s} / \Lambda$ with fiber $F$ and the natural $\mathbb{C}^{s}$-action on $F \times \mathbb{C}^{s}$ commutes with $\Lambda$ inducing a locally free action on $X$. We say that $X$ is a suspension over $\mathbb{T}^{s}$. It is proved in [29, Theorem 3.19] that $X$ is a Kähler manifold if and only if $F$ is Kähler and there are integers $n_{i}$ for $i=1, \ldots, s$ such that $f_{i}^{n_{i}} \in \operatorname{Aut}_{\mathbb{C}}^{0}(F)$. 
Remark 2.5. The above characterizations of Kähler manifolds that are principal torus bundles or suspensions over tori stated in the last two examples rely on a criterion, due to Blanchard [5], for deciding if the total space of a fibration is a Kähler manifold. It is not known if Blanchard's criterion can be extended to the class $\mathcal{C}$. Therefore we are not able to formulate such kind of characterizations for Fujiki manifolds. This is the main difficulty to extend the structure theorems in Section 7 to manifolds in the class $\mathcal{C}$.

From now on $X$ will denote a given compact manifold in the class $\mathcal{C}$ and we will use the notation introduced in Section 1 . We denote $b_{1}(X)=2 p$. We also assume from now on that $s=\operatorname{dim}_{\mathbb{C}} \mathfrak{h} / \mathfrak{h}_{1}$ is strictly positive and we fix a subalgebra $\mathfrak{a} \subset \mathfrak{h}_{X}$ with $\mathfrak{h}=\mathfrak{h}_{X}^{1} \oplus \mathfrak{a}$ as well as a basis $v_{1}, \ldots, v_{s}$ of $\mathfrak{a}$. We denote by $\varpi$ the locally free $\mathbb{C}^{S}$-action defined by the choice of the basis, and by $\mathcal{F}$ the induced foliation.

It follows from Theorem 1.2 that there are holomorphic 1-forms $\alpha^{1}, \ldots, \alpha^{s}$ such that $\alpha^{i}\left(v_{i}\right)=1$ and $\alpha^{j}\left(v_{i}\right)=0$ if $j \neq i$. The forms $\alpha^{i}$ generate the cotangent bundle $T^{*} \mathcal{F}$ of $\mathcal{F}$ at each point and we assume that they have also been fixed. The distribution $\operatorname{ker} \alpha^{1} \cap$ $\cdots \cap \operatorname{ker} \alpha^{s}$ is integrable and defines a holomorphic foliation $\mathcal{G}$ that depends on the choice of $\alpha^{1}, \ldots, \alpha^{s}$. This foliation is transverse and complementary to $\mathcal{F}$, and also invariant by the action of $\mathbb{C}^{s}$. Therefore, we have

Proposition 2.6. A foliation $\mathcal{F}$ on a compact manifold $X$ in the class $\mathcal{C}$ defined by a locally free $\mathbb{C}^{S}$-action $\varpi$ admits a holomorphic foliation $\mathcal{G}$, which is transverse and complementary to $\mathcal{F}$, and invariant by the action $\varpi$.

Remarks 2.7. (a) This proposition implies in particular that a principal torus bundle whose total space is a manifold in the class $\mathcal{C}$ is necessarily flat (cf. Example 2.3).

(b) Notice that all the leaves of $\mathcal{G}$ are biholomorphic. If the leaves of $\mathcal{G}$ are compact they define a fibration of $X$ over a complex torus of dimension $s$ and $X$ is a suspension.

We denote by $\nu \mathcal{F}$ the normal bundle of the foliation $\mathcal{F}$, i.e. $\nu \mathcal{F}=T X / T \mathcal{F}$. The existence of the $\mathbb{C}^{S}$-invariant foliation $\mathcal{G}$ implies that, at each point of $X$, we can find local coordinates $\left(t^{1}, \ldots, t^{s}, z^{1}, \ldots, z^{n-s}\right)$ with the properties

$$
v_{i}=\frac{\partial}{\partial t^{i}} \quad \text { and } \quad \alpha^{i}=d t^{i} .
$$

In these coordinates $\mathcal{F}$ is defined by $z^{k}=$ const and $\mathcal{G}$ by $t^{i}=$ const. As a consequence, we obtain:

Proposition 2.8. There is a natural isomorphism between the canonical bundle $K_{X}$ of $X$ and the determinant det $v^{*} \mathcal{F}$ of the conormal bundle $v^{*} \mathcal{F}$ of $\mathcal{F}$.

Proof. If $\left(t_{i}^{1}, \ldots, t_{i}^{s}, z_{i}^{1}, \ldots, z_{i}^{n-s}\right)$ are local coordinates satisfying (4) and we define $\eta_{i}=\alpha^{1} \wedge \cdots \wedge \alpha^{s} \wedge d z_{i}^{1} \wedge \cdots \wedge d z_{i}^{n-s}$, then $\eta_{j}=g_{j i} \eta_{i}$ with

$$
g_{j i}=\operatorname{det}\left(\frac{\partial z_{j}^{k}}{\partial z_{i}^{l}}\right) .
$$

Hence the cocycle $\left(g_{j i}\right)$ defines the line bundle $K_{X}$ as well as det $v^{*} \mathcal{F}$.

We then obtain: 
Corollary 2.9. Assume that $X$ is a suspension $F \times_{\Lambda} \mathbb{C}^{s}$ over a torus $T=\mathbb{C}^{s} / \Lambda$. Then $\operatorname{kod}(X)=\operatorname{kod}(F)$ and $c_{1}(F)=\iota^{*} c_{1}(X)$ where $\iota: F \hookrightarrow X$ is the identification of $F$ with a fiber of the projection $X \rightarrow T$.

Proof. Notice that the tangent bundle $T F$ of $F$ is canonically isomorphic to the restriction of $\nu \mathcal{F}$ to $F$. As the Kodaira dimension is invariant by finite coverings we can assume, using [29, Theorem 3.19], that the monodromy of the suspension has values in $\operatorname{Aut}_{\mathbb{C}}^{0}(F)$. By Proposition 2.8 there are isomorphisms $K_{X}^{\otimes m} \cong\left(\operatorname{det} v^{*} \mathcal{F}\right)^{\otimes m}$ for every $m \in \mathbb{Z}$. Consequently, the spaces $H^{0}\left(X, K_{X}^{\otimes m}\right)$ are naturally identified with the subspaces of $H^{0}\left(F,\left(\operatorname{det} v^{*} \mathcal{F}\right)^{\otimes m}\right)=H^{0}\left(F, K_{F}^{\otimes m}\right)$ invariant by the monodromy. Ueno proved [32, Corollary 14.8] that $\operatorname{Aut}_{\mathbb{C}}^{0}(F)$ acts trivially on the pluricanonical section spaces $H^{0}\left(F, K_{F}^{\otimes m}\right)$. Therefore $h^{0}\left(X, K_{X}^{\otimes m}\right)=h^{0}\left(F, K_{F}^{\otimes m}\right)$ for every $m$.

The relation between the first Chern classes follows from the adjunction formula.

Let $\Omega_{X}^{k}$ be the sheaf of germs of holomorphic k-forms on $X$. As remarked above, $p=$ $b_{1}(X) / 2 \geq s$. We can choose holomorphic 1 -forms $\beta^{1}, \ldots, \beta^{p-s}$ on $X$ such that

$$
\left\{\alpha^{1}, \ldots, \alpha^{s}, \beta^{1}, \ldots, \beta^{p-s}\right\}
$$

is a basis of $H^{0}\left(X, \Omega_{X}^{1}\right)$ and $\beta^{j}\left(v_{i}\right)=0$ for $i=1, \ldots, s$ and $j=1, \ldots, p-s$. Since $\beta^{j}$ are closed, they are in fact basic forms with respect to $\mathcal{F}$. We recall that a differential form $\gamma$ is said to be basic with respect to a foliation if $i_{w} \gamma=0$ and $i_{w} d \gamma=0$ for each local vector field $w$ tangent to the foliation. We shall denote by $\Omega_{X / \mathcal{F}}^{k}$ the subsheaf of $\Omega_{X}^{k}$ of those $k$-forms that are basic with respect to $\mathcal{F}$. The following decomposition of global holomorphic forms on $X$ will be useful.

Lemma 2.10. Each global holomorphic $k$-form $\gamma$ on $X$ can be written in a unique way as a sum

$$
\gamma=\sum_{0 \leq|I| \leq \min (k, s)} \alpha^{I} \wedge \gamma^{I}
$$

with $I=\left(i_{1}, \ldots, i_{m}\right), 1 \leq i_{1}<\cdots<i_{m} \leq s$ and $|I|=m$, and where $\alpha^{I}=\alpha^{i_{1}} \wedge \cdots \wedge$ $\alpha^{i_{m}}$ and $\gamma^{I}$ is a holomorphic $(k-m)$-form basic with respect to $\mathcal{F}$. In particular

$$
H^{0}\left(X, \Omega_{X}^{k}\right) \cong \bigoplus_{i=0}^{\min (k, s)} \bigwedge^{i}\left\langle\alpha^{1}, \ldots, \alpha^{s}\right\rangle_{\mathbb{C}} \otimes H^{0}\left(X, \Omega_{X / \mathcal{F}}^{k-i}\right)
$$

Proof. Let $\gamma$ be a global holomorphic $k$-form on $X$ and set $\ell=\min (k, s)$. For each $I=\left(i_{1}, \ldots, i_{m}\right)$ with $m \leq k$ and $1 \leq i_{1}<\cdots<i_{m} \leq s$, and a given $k$-form $\omega$, we denote $\omega^{I}=i_{v_{m}} \circ \cdots \circ i_{v_{i_{1}}} \omega$. We put $\gamma_{0}=\gamma$ and we define $\gamma_{1}, \ldots, \gamma_{\ell}$ recursively by

$$
\gamma_{m+1}=\gamma_{m}-\sum_{|I|=\ell-m} \alpha^{I} \wedge \gamma_{m}^{I}
$$

Notice here that the $(k-\ell+m)$-forms $\gamma_{m}^{I}$ are holomorphic global forms and thus they are closed. Moreover, by construction they satisfy $i_{v} \gamma_{m}^{I}=0$ for each vector $v$ tangent to $\mathcal{F}$. 
Therefore they are basic with respect to $\mathcal{F}$ and

$$
\gamma=\sum_{m=1}^{\ell} \sum_{|I|=m} \alpha^{I} \wedge \gamma_{\ell-m}^{I}+\gamma_{\ell}
$$

is the required decomposition.

\section{Projective manifolds with nonvanishing tangent fields}

In this section we will assume that $X$ is a complex projective manifold. Our study in this algebraic context becomes a continuation of the classical study of complex projective manifolds with holomorphic tangent vector fields, which led to their birational classification summarized in Theorem 0.1. The Albanese mapping and Poincaré's reducibility theorem lead to a biholomorphic classification of these manifolds which is simpler than in the Kähler case.

In view of Theorem 1.2 and Remark 1.5 about the structure of the Lie algebra of holomorphic tangent vector fields, the existence of nonvanishing tangent vector fields is equivalent to the existence of a holomorphic locally free $\mathbb{C}^{r}$-action on the manifold. We will work directly with these locally free actions. The basic fact beyond the classical theory is:

Proposition 3.1. Let $X$ be a complex projective manifold, admitting a locally free holomorphic $\mathbb{C}^{r}$-action. Then $X$ is a suspension $F \times{ }_{\Lambda} \mathbb{C}^{s}$ over an Abelian variety of dimension $s \geq r$, with fiber a connected projective manifold $F$.

Proof. As $X$ is closed Kähler, the $\mathbb{C}^{r}$-action can be extended to a locally free holomorphic action of maximal rank $s=\operatorname{dim}_{\mathbb{C}} T_{X}=\operatorname{dim} \mathfrak{h}_{X} / \mathfrak{h}_{X}^{1}$ as we have recalled in Section 1.

The Albanese torus $\operatorname{Alb}(X)$ of $X$ is an Abelian variety, therefore the subtorus $T_{X} \subset$ $\operatorname{Alb}(X)$ in the Fujiki-Lieberman structure Theorem 1.2 is also an Abelian variety, and by Poincaré's Reducibility Theorem (see $[4, \S 5.3]$ ) it has a complementary Abelian subvariety $Z$ such that $T_{X} \cap Z$ is finite, $T_{X}+Z=\operatorname{Alb}(X)$, and the addition law induces an isogeny $c: \operatorname{Alb}(X) \rightarrow T_{X} \times Z$.

The composition of the Albanese morphism of $X$ with this isogeny and the natural projection

$$
X \stackrel{\phi}{\rightarrow} \operatorname{Alb}(X) \stackrel{c}{\rightarrow} T_{X} \times Z \stackrel{p_{1}}{\rightarrow} T_{X}
$$

maps the tangent subspace of $X$ generated by the $\mathbb{C}^{S}$-action isomorphically into the tangent space of $T_{X}$. Consequently, $X$ is a suspension over $T_{X}$, with parallel transport given by the $\mathbb{C}^{S}$-action and possibly disconnected fibers. The Stein factorization of $X \rightarrow T_{X}$ is thus unramified, and yields a suspension $X \rightarrow N_{X}$ with connected fiber $F$, over an Abelian variety $N_{X}$ isogenous to $T_{X}$.

If $X$ is not ruled, it follows from Theorems 0.1 and 1.2 that $\operatorname{Aut}_{\mathbb{C}}^{0}(X)$ is an Abelian variety of dimension $s=\operatorname{dim} \mathfrak{h}_{X}$, isogenous to $N_{X}$. The suspension morphism $X \rightarrow N_{X}$ shows that the natural action of $\operatorname{Aut}_{\mathbb{C}}^{0}(X)$ on $X$ is holomorphically injective, and the above 
quoted theorems make Proposition 3.1 essentially equivalent to Carrell's classification theorem for such actions of Abelian varieties (cf. [10, Thm. 8]).

The classification of projective manifolds with locally free holomorphic $\mathbb{C}^{s}$-actions follows from Proposition 3.1 and Theorem 1.8. Putting them together yields:

Theorem 3.2. Let $X$ be a complex projective manifold admitting a locally free holomorphic $\mathbb{C}^{r}$-action.

(a) If $X$ is not uniruled, it is a quotient $(F \times T) / \Gamma$, with $T$ an Abelian variety of dimension $s \geq r, F$ a projective manifold with no holomorphic tangent vector fields and $\operatorname{kod}(F)=\operatorname{kod}(X)$, and $\Gamma$ a finite Abelian group of biholomorphisms operating freely on $F \times T$.

(b) If $X$ is uniruled, it admits a finite, Abelian, étale cover $X^{\prime}$ which is a suspension over an Abelian variety $T$ of dimension $s \geq r$, with uniruled fiber $F$ such that any holomorphic tangent vector field on $F$ has zeros. The monodromy of the suspension $X^{\prime} \rightarrow T$ has values in the group $\operatorname{Aut}_{\mathbb{C}}^{0}(F)$.

Remark 3.3. This theorem proves the algebraic part of Theorem 0.3. Its proof mirrors that of Proposition 6.5 which deals with the general case of Kähler manifolds. As examples in Section 7 show, the fact that Poincarés reducibility theorem is no longer valid in the Kähler setting forces the introduction of deformations of the complex structure.

Proof. We may assume that the locally free action on $X$ is of maximal rank $s$. By Proposition 3.1, $X$ is a suspension over an $s$-dimensional Abelian variety $N_{X}$, isogenous to $T_{X}$, and with fiber a connected projective manifold $F_{1}$.

Let the suspension structure on $X$ be given by $N_{X}=\mathbb{C}^{s} / \Lambda$, with $\Lambda=\left\langle\tau_{1}, \ldots, \tau_{2 s}\right\rangle$ a cocompact lattice defining the torus $N_{X}, \rho: \Lambda \rightarrow \operatorname{Aut}_{\mathbb{C}}(F)$ the monodromy of the suspension, and $f_{i}=\rho\left(\tau_{i}\right)$ the generators of the monodromy.

By 2.4, the fact that the total space $X$ of the suspension is Kähler implies that all the monodromy automorphisms have a finite power $f_{i}^{n_{i}} \in \operatorname{Aut}_{\mathbb{C}}^{0}(F)$, i.e. there is a finite étale Abelian covering $T_{1} \rightarrow N_{X}$ such that the pullback of $X$ over $T_{1}$ is a finite étale Abelian covering $X_{1} \rightarrow X$, and also a suspension over $T_{1}$ with fiber $F_{1}$ and monodromy automorphisms in $\operatorname{Aut}_{\mathbb{C}}^{0}\left(F_{1}\right)$.

If $F_{1}$ has no nonvanishing holomorphic tangent vector fields it can be checked that we have reached the desired cover:

(i) $\operatorname{kod}\left(F_{1}\right)=\operatorname{kod}(X)$ by Corollary 2.9.

(ii) If $X$ is not uniruled, neither is $X_{1}$ nor $F_{1}$. Indeed, as all the fibers of $X_{1} \rightarrow T_{1}$ are isomorphic, if $F_{1}$ were uniruled there would be a rational curve passing through each given point of $X_{1}$. Now Fujiki's Theorem 1.8 implies that $\mathfrak{h}_{F_{1}}^{1}=0$. Therefore the suspension monodromy has values in $\operatorname{Aut}_{\mathbb{C}}^{0}\left(F_{1}\right)=\{\mathrm{Id}\}$, i.e. $X_{1}$ is biholomorphic to $F_{1} \times T_{1}$.

(iii) If $X$ is uniruled so is its étale cover $X_{1}$. Since rational curves map to points in the suspension basis $T_{1}$, the fiber $F_{1}$ is uniruled as well. 
If $F_{1}$ has nonvanishing holomorphic tangent vector fields, we apply an iterative process: let $\mathfrak{h}_{F_{1}}$ be the Lie algebra of holomorphic vector fields in $F_{1}$, and apply Proposition 1.6 to choose an Abelian subalgebra $\mathfrak{a}_{F_{1}}$ corresponding to a $\mathbb{C}^{r}$-action of maximal rank in $F_{1}$, such that $\mathfrak{a}_{F_{1}}$ is central in $\mathfrak{h}_{F_{1}}$.

As the monodromy of the suspension $X_{1} \rightarrow T_{1}$ lies in $\operatorname{Aut}_{\mathbb{C}}^{0}\left(F_{1}\right)$ it must commute with the central algebra $\mathfrak{a}_{F_{1}}$. Therefore its tangent vector fields may be extended by parallel transport from the fiber $F_{1}$ to the suspension total space $X_{1}$ as nonvanishing tangent vector fields. These extensions, to be denoted $\left\langle v_{1}, \ldots, v_{r}\right\rangle$, commute with the horizontal vector fields $\left\langle h_{1}, \ldots, h_{s}\right\rangle$ which are the lifts to $X_{1}$ of $\mathfrak{h}_{T_{1}}=H^{0}\left(T_{1}, \Theta_{T_{1}}\right)$ defining the suspension's parallel transport. Thus $\mathfrak{a}_{X_{1}}=\left\langle v_{1}, \ldots, v_{r}, h_{1}, \ldots, h_{s}\right\rangle$ is an Abelian subalgebra of $\mathfrak{h}_{X_{1}}$, spanned by $r+s$ linearly independent nonvanishing vector fields. It has maximal rank among such algebras: the algebra $\mathfrak{h}_{X_{1}}$ is the direct sum

$$
\mathfrak{h}_{X_{1}}=\left\langle h_{1}, \ldots, h_{s}\right\rangle \oplus H^{0}\left(X_{1}, \mathcal{V} \text { ert }\right)
$$

where $\mathcal{V}$ ert is the sheaf spanned by the vector fields vertical with respect to the projection $X_{1} \rightarrow T_{1}$. As the horizontal fields $h_{1}, \ldots, h_{s}$ are central in $\mathfrak{h}_{X_{1}}$, the morphism $H^{0}\left(X_{1}, \mathcal{V}\right.$ ert $) \rightarrow \mathfrak{h}_{F_{1}}$ given by restriction to a fiber is injective. This induces a natural inclusion of Lie algebras

$$
\mathfrak{h}_{X_{1}}=\left\langle h_{1}, \ldots, h_{s}\right\rangle \oplus H^{0}\left(X_{1}, \mathcal{V} e r t\right) \subset\left\langle h_{1}, \ldots, h_{s}\right\rangle \oplus \mathfrak{h}_{F_{1}}
$$

If there existed an Abelian Lie subalgebra $\tilde{\mathfrak{a}} \subset \mathfrak{h}_{X_{1}}$ formed by nonvanishing tangent fields, with $\operatorname{dim} \tilde{\mathfrak{a}}>r+s$, then its inclusion in $\left\langle h_{1}, \ldots, h_{s}\right\rangle \oplus \mathfrak{h}_{F_{1}}$ would have an intersection with $\mathfrak{h}_{F_{1}}$ of dimension $>r$. This contradicts the maximality of $\mathfrak{a}_{F_{1}}$.

By Proposition 3.1, the algebra $\mathfrak{a}_{X_{1}}$ determines a suspension $X_{1} \rightarrow N_{1}$, over an $(r+s)$-dimensional Abelian variety, with a connected fiber $F_{2}$. Again by 2.4 , the monodromy automorphisms of this suspension have a finite power in $\operatorname{Aut}_{\mathbb{C}}^{0}\left(F_{2}\right)$. Consequently, there is a finite étale Abelian covering $T_{2} \rightarrow N_{1}$ such that the pullback $X_{2}$ of $X_{1}$ over $T_{2}$ is a suspension with fiber $F_{2}$ and monodromy in $\operatorname{Aut}_{\mathbb{C}}^{0}\left(F_{2}\right)$.

The induced map $X_{2} \rightarrow X_{1}$ is also a finite étale Abelian cover. Moreover, the composition of coverings $X_{2} \rightarrow X_{1} \rightarrow X$ is still regular and Abelian. The reason for its regularity is that the automorphisms of the cover $X_{1} \rightarrow X$ are realized by integration up to time 1 in $X_{1}$ of suitable linear combinations of the horizontal fields $h_{1}, \ldots, h_{s}$. These tangent fields have become parallel transport vector fields of the suspension $X_{1} \rightarrow T_{1}$ by our choice of algebra $\mathfrak{h}_{X_{1}}$, and the isogenous base change $T_{2} \rightarrow T_{1}$ lifts canonically to $X_{2}$ the vector fields $h_{1}, \ldots, h_{s}$, thus also the integration up to time 1 of their linear combinations. The Abelianity of the cover follows from the fact that the vector fields $v_{1}, \ldots, v_{r}, h_{1}, \ldots, h_{s}$ commute in $X_{1}$, hence also in $X_{2}$, and the automorphisms of both covers $X_{1} \rightarrow X, X_{2} \rightarrow X_{1}$ are defined by integration up to time 1 of suitable linear combinations of them.

The iteration step concludes here. We have obtained a suspension $X_{2} \rightarrow T_{2}$, with $X_{2}$ a finite, Abelian étale cover of $X$, fiber $F_{2}$ with $\operatorname{kod}\left(F_{2}\right)=\operatorname{kod}\left(X_{2}\right)=\operatorname{kod}(X)$, and monodromy in $\operatorname{Aut}_{\mathbb{C}}^{0}\left(F_{2}\right)$. 
Repetition of the above procedure yields a sequence of suspensions $X_{i} \rightarrow T_{i}$ with fiber $F_{i}$, again with $X_{i}$ Abelian étale covers of $X, \operatorname{kod}\left(F_{i}\right)=\operatorname{kod}(X)$ and monodromy in $\operatorname{Aut}_{\mathbb{C}}^{0}\left(F_{i}\right)$. As the dimension of the basis tori $T_{2}, T_{3}, \ldots$ grows strictly the iterative process must reach a final suspension $X_{f} \rightarrow T_{f}$ such that its fiber $F_{f}$ does not have any nonvanishing vector field, i.e. no holomorphic locally free $\mathbb{C}^{r}$-action.

If $X$ is not uniruled neither is $X_{f}$ nor $F_{f}$. By Fujiki's Theorem 1.8 we have $\mathfrak{h}_{F_{f}}^{1}=0$. Hence the fiber $F_{f}$ cannot have any tangent vector field, so $\operatorname{Aut}_{\mathbb{C}}^{0}\left(F_{f}\right)=\{\mathrm{Id}\}$ and the trivial suspension $X_{f}=F_{f} \times T_{f}$ has been reached.

If $X$ is uniruled, so are all its étale covers up to $X_{f}$, and the fiber $F_{f}$ as well because rational curves in $X_{f}$ must map to a point in $T_{f}$.

We can use the structure theorem 3.2 to classify algebraic manifolds with sufficiently many vector fields and to establish for them Ueno's conjecture 0.4. This is done in Corollaries 8.9 and 8.10 below as specialization of the arguments in the Kähler case.

\section{Tangential deformations of locally free holomorphic actions}

Throughout this section, $X$ will be an arbitrary compact complex manifold, i.e. not necessarily Kähler or belonging to the Fujiki class. Let us assume that $X$ is endowed with a holomorphic action $\varpi: G \times X \rightarrow X$, where $G$ is a connected complex Lie group. Such an action is defined by a representation

$$
\rho: G \rightarrow \operatorname{Aut}_{\mathbb{C}}(X)
$$

from $G$ into the group $\operatorname{Aut}_{\mathbb{C}}(X)$ of holomorphic automorphisms of $X$. When $\varpi$ is locally free, i.e. it has discrete isotropy groups, then it induces a holomorphic foliation $\mathcal{F}$ on $X$ whose leaves are the orbits of the action.

Let $\mathcal{F}^{\text {tr }}$ denote the transversely holomorphic foliation obtained from $\mathcal{F}$ by forgetting the complex structure along the leaves. One can consider deformations of the holomorphic foliation $\mathcal{F}$ that keep fixed its transversal type; that is, holomorphic deformations $\mathcal{F}_{r}$ of $\mathcal{F}$ such that the transversely holomorphic foliation $\mathcal{F}_{r}^{\operatorname{tr}}$ coincides with $\mathcal{F}^{\operatorname{tr}}$. This type of deformations were studied in [19] and [18], where they are called $f$-deformations. In [19], Gómez-Mont studies the space of infinitesimal $f$-deformations of holomorphic foliations, which is naturally identified to $H^{1}(X, T \mathcal{F})$, i.e. the first cohomology group of $X$ with values in the tangent bundle $T \mathcal{F}$ of $\mathcal{F}$. The existence of a versal or Kuranishi space for $f$-deformations, i.e. a germ of analytic space $\left(\mathcal{K}^{f}, 0\right)$ parametrizing a versal family of $f$-deformations of $\mathcal{F}$, is proved in [18]. The tangent space to $\left(\mathcal{K}^{f}, 0\right)$ at 0 is $H^{1}(X, T \mathcal{F})$.

Families of $f$-deformations of $\mathcal{F}$ can be viewed as unfoldings of $\mathcal{F}$, that is, families of complex structures on $X$ for which $\mathcal{F}^{\text {tr }}$ becomes holomorphic. More precisely, a family of $f$-deformations of $\mathcal{F}$ parametrized by a germ of analytic space $\left(R, r_{0}\right)$ is given by a proper and flat morphism $\pi: X_{R} \rightarrow R$, an identification $\iota: X \hookrightarrow X_{R}$ of $X$ with the fiber $X_{r_{0}}=\pi^{-1}\left(r_{0}\right)$ and a holomorphic foliation $\mathcal{F}_{R}$ on $X_{R}$ of the same codimension as $\mathcal{F}$, 
which is transverse to the projection $\pi$ and such that the restriction $\mathcal{F}_{r_{0}}$ of $\mathcal{F}_{R}$ to $X \equiv X_{r_{0}}$ coincides with $\mathcal{F}$. Such a family of $f$-deformations will be denoted by $\left(X_{R}, \iota, \pi, \mathcal{F}_{R}\right)$.

Notice however that $f$-deformations $\mathcal{F}_{r}$ of $\mathcal{F}$ need not be equivariant, in the sense that $\mathcal{F}_{r}$ are not necessarily defined by an action. For this reason we give the following definition, which is inspired by the notion of equivariant deformations of complex manifolds introduced by Cathelineau [13].

Definition 4.1. Let $X$ be a compact complex manifold endowed with a locally free holomorphic action $\varpi: G \times X \rightarrow X$ and let $\mathcal{F}$ be the foliation defined by $\varpi$. By a family of tangential deformations of $\varpi$ we mean a family of $f$-deformations $\left(X_{R}, \iota, \pi, \mathcal{F}_{R}\right)$ of $\mathcal{F}$ and a holomorphic action $\varpi_{R}$ of $G$ on $X_{R}$ with the following properties:

(i) $\varpi_{R}$ extends the action $\varpi$ of $G$ on $X \equiv \iota(X)=X_{r_{0}}$,

(ii) $\varpi_{R}$ preserves the fibers of $\pi$, and

(iii) the orbits of the action are tangent to $\mathcal{F}_{R}$.

Then $\varpi_{R}$ induces a locally free $G$-action $\varpi_{r}$ on $X_{r}$ for each $r \in R$ and the leaves of $\mathcal{F}_{r}=\left.\mathcal{F}\right|_{X_{r}}$ are the orbits of $\varpi_{r}$.

Such a family will be denoted by $\left(X_{R}, \iota, \pi, \varpi_{R}\right)$ and each pair $\left(X_{r}, \varpi_{r}\right)$ with $r \in R$ will be called a tangential deformation of $\left(X_{0}, \varpi_{0}\right) \cong(X, \varpi)$. We will just write $\left(X_{R}, \varpi_{R}\right)$ instead of $\left(X_{R}, \iota, \pi, \varpi_{R}\right)$ when there is no danger of confusion.

Definition 4.2. Two families $\left(X_{R}, \iota, \pi, \varpi_{R}\right)$ and $\left(X_{R}^{\prime}, \iota^{\prime}, \pi^{\prime}, \varpi_{R}^{\prime}\right)$ of tangential deformations of $(X, \varpi)$, parametrized by the same germ of analytic space $R$, are said to be $i s o$ morphic if there is a $G$-equivariant biholomorphism $\phi: X_{R} \rightarrow X_{R}^{\prime}$ satisfying $\phi \circ \iota=\iota^{\prime}$ and $\pi^{\prime} \circ \phi=\pi$.

A family of tangential deformations $\left(X_{R}, \varpi_{R}\right)$ of $(X, \varpi)$ is called versal if it has the following property: for any other family $\left(X_{S}, \varpi_{S}\right)$ of tangential deformations of $(X, \varpi)$ there is an analytic morphism of germs of analytic spaces $\varphi: S \rightarrow R$ such that

(i) the pull-back family $\left(\varphi^{*}\left(X_{R}\right), \varphi^{*}\left(\varpi_{R}\right)\right)$ parametrized by $S$ is isomorphic to $\left(X_{S}, \varpi_{S}\right)$, and

(ii) the tangent map $d_{s_{0}} \varphi$ of $\varphi$ at the distinguished point $s_{0}$ of $S$ is unique.

If such a versal family exists then it is unique up to isomorphism.

Notice now that the sheaf of germs of holomorphic vector fields tangent to $\mathcal{F}$ is a $G$-sheaf and therefore we can consider the equivariant cohomology of $X$ with values in the tangent bundle $T \mathcal{F}$ of $\mathcal{F}$, which we denote by $H_{G}^{*}(X, T \mathcal{F})$. Let $\Omega^{0, q}(X, T \mathcal{F})$ be the Fréchet space of $(0, q)$-forms on $X$ with values in $T \mathcal{F}$ and denote by $C_{h}^{p}\left(G, \Omega^{0, q}(X, T \mathcal{F})\right)$ the space of holomorphic $p$-cochains with values in the $G$-module $\Omega^{0, q}(X, T \mathcal{F})$. Then $H_{G}^{*}(X, T \mathcal{F})$ is the cohomology of the double complex

$$
\left(\left(C_{h}^{p}\left(G, \Omega^{0, q}(X, T \mathcal{F})\right)\right)_{p, q}, \delta, \bar{\partial}\right)
$$

where $\delta$ denotes the Eilenberg-MacLane coboundary operator and $\bar{\partial}$ is the usual deltabar operator. As usual, infinitesimal tangential deformations of $(X, \varpi)$ can be defined as 
isomorphism classes of families parametrized by the double point $D=\left\{*, \mathbb{C}(t) /\left(t^{2}\right)\right\}$. The space of infinitesimal deformations is naturally identified to $H_{G}^{1}(X, T \mathcal{F})$. Moreover, to each family of tangential deformations $\left(X_{R}, \varpi_{R}\right)$ of $(X, \varpi)$ there is associated in a natural way a linear map

$$
\kappa: T_{r_{0}} R \rightarrow H_{G}^{1}(X, T \mathcal{F})
$$

which is called the Kodaira-Spencer map of the family (cf. [13]).

The following theorem states the existence of a versal family and it can be proved along the same lines as the corresponding statement for equivariant deformations of complex manifolds (cf. [13, Théorème 1 and Proposition 1]).

Theorem 4.3. Let $X$ be a compact complex manifold endowed with a locally free holomorphic action $\varpi: G \times X \rightarrow X$ and let $\mathcal{F}$ be the induced holomorphic foliation. Then there is a germ of analytic space $\left(\mathcal{K}_{G}^{t}, 0\right)$ parametrizing a versal family of tangential deformations of the action $\varpi$. The tangent space to $\mathcal{K}_{G}^{t}$ at 0 is isomorphic to $H_{G}^{1}(X, T \mathcal{F})$ and it is naturally identified to the space of infinitesimal tangential deformations of $\varpi$.

The following proposition is a general fact in deformation theory and can be deduced easily from the existence of the versal space of tangential deformations.

Proposition 4.4. Let $\left(X_{R}, \varpi_{R}\right)$ be a family of tangential deformations of $(X, \varpi)$ whose parameter space $R$ is smooth and such that the corresponding Kodaira-Spencer map $\kappa$ is an isomorphism. Then the family of deformations $\left(X_{R}, \varpi_{R}\right)$ is versal.

Associated to the double complex (7) there is a spectral sequence converging to $H_{G}^{*}(X, T \mathcal{F})$ and whose second term is $E_{2}^{p, q}=H_{h}^{p}\left(G, H^{q}(X, T \mathcal{F})\right)$. Here the index $h$ denotes cohomology of holomorphic cochains. In particular one has the following exact sequence that is useful in the computation of $H_{G}^{1}(X, T \mathcal{F})$ :

$$
\begin{aligned}
0 \rightarrow H_{h}^{1}\left(G, H^{0}(X, T \mathcal{F})\right) \rightarrow H_{G}^{1}(X, T \mathcal{F}) \stackrel{x}{\rightarrow} H^{1}(X, T \mathcal{F})^{G} & \\
& \rightarrow H_{h}^{2}\left(G, H^{0}(X, T \mathcal{F})\right) \rightarrow H_{G}^{2}(X, T \mathcal{F}) .
\end{aligned}
$$

\section{Tangential deformations of locally free $\mathbb{C}^{s}$-actions on Kähler and Fujiki manifolds}

The aim of this section is to construct the versal family of tangential deformations of a locally free $\mathbb{C}^{s}$-action $\varpi$ on a given compact Kähler or Fujiki manifold $X$ and to study the properties of that family.

With this purpose we consider the exact sequence (8) when $G$ is the Abelian group $\mathbb{C}^{s}$. In that case $T \mathcal{F}$ is trivial as a $G$-bundle. So $G=\mathbb{C}^{s}$ acts trivially on $H^{0}(X, T \mathcal{F}) \equiv \mathbb{C}^{s}$ and $H_{h}^{1}\left(G, H^{0}(X, T \mathcal{F})\right)$ is just the space of holomorphic group homomorphisms from $G$ into $\mathbb{C}^{s}$, that is, the space End $\mathbb{C}^{s}$ of $\mathbb{C}$-linear endomorphisms of $\mathbb{C}^{s}$. Moreover, $G=\mathbb{C}^{s}$ also acts trivially on $H^{1}(X, T \mathcal{F})=H^{1}\left(X, \mathcal{O}_{X}\right)^{s} \cong H^{0}\left(X, \Omega_{X}^{1}\right)^{s}$, where the last isomorphism is $\mathbb{C}$-antilinear. 
Therefore, the first terms of sequence (8) can be written

$$
0 \rightarrow \operatorname{End} \mathbb{C}^{s} \stackrel{\tau}{\rightarrow} H_{G}^{1}(X, T \mathcal{F}) \stackrel{\chi}{\rightarrow} H^{0}\left(X, \Omega_{X}^{1}\right)^{s} \rightarrow \cdots
$$

We will see below that the morphism $\chi$ is surjective and therefore $H_{G}^{1}(X, T \mathcal{F})$ is isomorphic to the direct sum End $\mathbb{C}^{s} \oplus H^{0}\left(X, \Omega_{X}^{1}\right)^{s}$. This motivates the construction of the family of tangential deformations of $(X, \varpi)$ that follows.

As above, $v_{1}, \ldots, v_{s}$ will denote the fundamental vector fields of the action $\varpi$ corresponding to the canonical basis of $\mathbb{C}^{s}, \alpha^{1}, \ldots, \alpha^{s}$ will be holomorphic 1-forms satisfying $\alpha^{i}\left(v_{j}\right)=\delta_{j}^{i}$, and $\left\{\alpha^{1}, \ldots, \alpha^{s}, \beta^{1}, \ldots, \beta^{p-s}\right\}$ a basis of $H^{0}\left(X, \Omega_{X}^{1}\right)$ where $\beta^{k}$ are basic forms. Although the vector fields $v_{i}$ are naturally associated to the action $\varpi$, the 1-forms $\alpha^{i}$ are not uniquely determined. If $\tilde{\alpha}^{i}$ is another choice then $\tilde{\alpha}^{i}-\alpha^{i}$ are basic forms, hence vanish on $T \mathcal{F}$.

We denote $\Xi=$ End $\mathbb{C}^{s} \oplus H^{0}\left(X, \Omega_{X}^{1}\right)^{s}$. Given an element $r=(C, \theta) \in \Xi$, where $\theta=\left(\theta^{1}, \ldots, \theta^{s}\right)$ and $\theta^{i}=\sum_{j=1}^{s} a_{j}^{i} \alpha^{j}+\sum_{k=1}^{p-s} b_{k}^{i} \beta^{k}$ with $a_{j}^{i}, b_{k}^{i} \in \mathbb{C}$, we set

$$
\alpha_{r}^{i}=\alpha^{i}+\overline{\theta^{i}}=\alpha^{i}+\sum_{j=1}^{s} \overline{a_{j}^{i}} \overline{\alpha^{j}}+\sum_{k=1}^{p-s} \overline{b_{k}^{i}} \overline{\beta^{k}} \quad \text { for } i=1, \ldots, s .
$$

We set $A=\left(a_{j}^{i}\right)$ and

$$
M_{A}=\left(\begin{array}{cc}
I & -A \\
-\bar{A} & I
\end{array}\right)
$$

Definition 5.1. Let $R_{0}$ be the open subset of $H^{0}\left(X, \Omega_{X}^{1}\right)^{s}$ of those elements $\theta=$ $\left(\theta^{1}, \ldots, \theta^{s}\right)$ for which $\operatorname{det} M_{A} \neq 0$ and denote by $R$ the open subset of $\Xi$ whose elements are the pairs $r=(C, \theta)$ satisfying

(i) $\operatorname{det} C \neq 0$, and

(ii) $\operatorname{det} M_{A} \neq 0$,

that is, $R=\mathrm{GL}(s, \mathbb{C}) \times R_{0}$. The set $R$ is the complement of an affine real algebraic variety and its tangent space at (id, 0) is naturally identified to $\Xi=$ End $\mathbb{C}^{s} \oplus H^{0}\left(X, \Omega_{X}^{1}\right)^{s}$.

The space $R$ will be the parameter space of the versal family of tangential deformations of $(X, \varpi)$. Moreover, when $X$ is a Kähler manifold, that family will be versal at each point of $R$.

Condition (ii) in the above definition implies that $\alpha_{r}^{1}, \ldots, \alpha_{r}^{s}$ are linearly independent at each point. Hence the forms $\alpha_{r}^{i}$ generate a real subbundle $Q_{r}$ of $T^{*} X^{\mathbb{C}}$. One has $Q_{r} \cap \nu^{*} \mathcal{F}=0$, so there are well defined complex valued smooth vector fields $w_{1}, \ldots, w_{s}$ that are tangent to $\mathcal{F}$ and determined by the conditions

$$
\alpha_{r}^{i}\left(w_{j}\right)=\delta_{j}^{i} \quad \text { and } \quad \alpha_{r}^{i}\left(\bar{w}_{j}\right)=0 .
$$

We set

$$
\tilde{w}_{i}=v_{i}-\sum_{j=1}^{s} a_{i}^{j} \bar{v}_{j} \quad \text { for } i=1, \ldots, s
$$


and we notice that

$$
\left\langle w_{1}, \ldots, w_{s}\right\rangle_{\mathbb{C}}=\left\langle\tilde{w}_{1}, \ldots, \tilde{w}_{s}\right\rangle_{\mathbb{C}} \cong \mathbb{C}^{s}
$$

Then we define

$$
v_{i}^{r}=C w_{i}
$$

In this situation one has

Proposition 5.2. Let an element $r=(C, \theta)$ of $R$ be given and set $N_{r}^{1,0}=Q_{r} \oplus v^{*} \mathcal{F}^{1,0}$. Then $T^{*} X^{\mathbb{C}}=N_{r}^{1,0} \oplus \overline{N_{r}^{1,0}}$ and the almost complex structure so defined is integrable, defining a complex structure $X_{r}$. Moreover, the vector fields $v_{i}^{r}$ are holomorphic and induce a locally free $\mathbb{C}^{S}$-action $\varpi_{r}$ on $X_{r}$, which is a tangential deformation of $\varpi$. This construction defines a family $\left(X_{R}, \varpi_{R}\right)$ of tangential deformations of $(X, \varpi)$ parametrized by $R$.

Proof. The condition $T^{*} X^{\mathbb{C}}=N_{r}^{1,0} \oplus \overline{N_{r}^{1,0}}$ is equivalent to $\operatorname{det} M_{A} \neq 0$. Since $\alpha_{r}^{i}=$ $\alpha^{i}+\bar{\theta}^{i}$ are closed, the almost complex structure on $X$ defined by $N_{r}^{1,0}$ is integrable and induces a complex structure $X_{r}$ by Newlander-Nirenberg's theorem.

Since $v_{1}, \ldots, v_{s}$ are holomorphic on $X$ we see, using (13), that the vector fields $w_{1}, \ldots, w_{s}$ commute with each other. It is also clear that $\tilde{w}_{i}$ are of type $(1,0)$ on $X_{r}$, and so are the vector fields $w_{i}$. It only remains to show that $w_{i}$ are in fact holomorphic on $X_{r}$.

Let $\left(t^{1}, \ldots, t^{s}, z^{1}, \ldots, z^{n-s}\right)$ be local coordinates satisfying (4). A local basis of vector fields of type $(1,0)$ on $X_{r}$ is given by

$$
\left\{\tilde{w}_{1}, \ldots, \tilde{w}_{s}, \frac{\partial}{\partial z^{1}}-\sum_{j=1}^{s} c_{1}^{j} \bar{v}_{j}, \ldots, \frac{\partial}{\partial z^{n-s}}-\sum_{j=1}^{s} c_{n-s}^{j} \bar{v}_{j}\right\},
$$

where $c_{l}^{j}=\sum_{k=1}^{n-s} b_{k}^{j} \beta^{k}\left(\partial / \partial z^{l}\right)$. Notice that the functions $c_{l}^{j}$ are holomorphic on $X_{r}$ and basic with respect to the foliation $\mathcal{F}$, that is, $c_{l}^{j}$ only depend on the coordinates $z^{1}, \ldots, z^{n-s}$. The vector fields $w_{i}$ are holomorphic if and only if the Lie brackets $\left[w^{i}, \bar{w}\right]$ are of type $(0,1)$ on $X_{r}$ for each local vector field $w$ of type $(1,0)$ on $X_{r}$. But this is a straightforward computation using the local basis of $T X_{r}^{1,0}$ given above.

Remarks 5.3. (a) Notice that the almost complex structure on $X$ by the decomposition $T^{*} X^{\mathbb{C}}=N_{r}^{1,0} \oplus \overline{N_{r}^{1,0}}$ is real-analytic.

(b) The matrix $C$ in $r=(C, \theta)$ corresponds to a choice of a basis of fundamental vector fields of the action. Hence, if $r=(C, \theta)$ and $r^{\prime}=\left(C^{\prime}, \theta\right)$ with $C^{\prime} \neq C$ then the complex manifolds $X_{r}$ and $X_{r^{\prime}}$ are identical, as also are the holomorphic foliations $\mathcal{F}_{r}$ and $\mathcal{F}_{r^{\prime}}$, although the actions $\varpi_{r}$ and $\varpi_{r^{\prime}}$ are different.

(c) One can consider what, in principle, could be a more general class of tangential deformations of the action, namely those determined by the global 1-forms $\tilde{\alpha}^{\ell}$ on $X$ defined as

$$
\tilde{\alpha}^{\ell}=\alpha^{\ell}+\sum \overline{e_{j}^{\ell}} \overline{\alpha^{j}}+\sum \overline{f_{k}^{\ell}} \overline{\beta^{k}}+\sum g_{j}^{\ell} \alpha^{j}+\sum h_{k}^{\ell} \beta^{k}
$$


where the coefficients $e_{j}^{\ell}, f_{k}^{\ell}, g_{j}^{\ell}, h_{k}^{\ell}$ are complex numbers. In fact, if these coefficients are small enough then there are smooth vector fields uniquely defined by the conditions $\tilde{\alpha}^{\ell}\left(\tilde{v}_{j}\right)=\delta_{j}^{\ell}$ and $\overline{\tilde{\alpha}^{\ell}}\left(\tilde{v}_{j}\right)=0$. Moreover, if we denote by $\tilde{Q}$ the real subbundle of $T^{*} X^{\mathbb{C}}$ generated by the 1 -forms $\tilde{\alpha}^{\ell}$, then $N^{1,0}=\tilde{Q} \oplus v^{*} \mathcal{F}^{1,0}$ defines an integrable almost complex structure and the vector fields $\tilde{v}_{j}$ are holomorphic on the new complex manifold $\tilde{X}$.

Notice however that this complex structure and the vector fields $\tilde{v}_{j}$ are the same as the ones determined by the 1 -forms

$$
\alpha^{\ell}+\sum \overline{e_{j}^{\ell}} \overline{\alpha^{j}}+\sum \overline{f_{k}^{\ell}} \overline{\beta^{k}}+\sum g_{j}^{\ell} \alpha^{j} .
$$

Furthermore, $\tilde{X}$ endowed with the $\mathbb{C}^{s}$-action defined by the vector fields $\tilde{v}_{j}$ can be identified with a suitable $\left(X_{r}, \varpi_{r}\right)$ in the family constructed above. More precisely, if we denote by $D$ the invertible matrix $\left(\delta_{j}^{\ell}+g_{j}^{\ell}\right)$ then $\tilde{X}$ coincides with $X_{r}$, where $r=$ $\left(C,\left(\theta^{1}, \ldots, \theta^{s}\right)\right)$ is determined by $C=\left(c_{m}^{i}\right)=D^{-1}$ and $\overline{\theta^{i}}=\sum \overline{a_{j}^{i}} \overline{\alpha^{j}}+\sum \overline{b_{k}^{i}} \overline{\beta^{k}}$ with $\overline{a_{j}^{i}}=\sum c_{m}^{i} \overline{e_{j}^{m}}$ and $\overline{b_{j}^{i}}=\sum c_{m}^{i} \overline{f_{j}^{m}}$. Therefore this construction does not provide more general deformations of $(X, \varpi)$. In fact it is proved in the following theorem that $\left(X_{R}, \varpi_{R}\right)$ is the versal family of tangential deformations of $(X, \varpi)$. In particular the family is complete and it contains all the small tangential deformations of $(X, \varpi)$ up to isomorphism.

(d) It follows from the above remark that the family $\left(X_{R}, \varpi_{R}\right)$ does not depend on the choice of the 1 -forms $\alpha^{i}$ satisfying $\alpha^{i}\left(v_{j}\right)=\delta_{j}^{i}$. Therefore that family is naturally associated to $(X, \varpi)$.

Remark 5.4. Let $\varpi^{\prime}$ be a locally free $\mathbb{C}^{S}$-action on a compact complex manifold $X^{\prime}$ and assume that $\left(X^{\prime}, \varpi^{\prime}\right)$ coincides with a pair $\left(X_{r}, \varpi_{r}\right)$ in the family $\left(X_{R}, \varpi_{R}\right)$. Then $(X, \varpi)$ coincides with an element of the family $\left(X_{R}^{\prime}, \varpi_{R}^{\prime}\right)$ associated to $\left(X^{\prime}, \varpi^{\prime}\right)$. Therefore the original action $\varpi: G \times X \rightarrow X$ is also a tangential deformation of $\varpi_{r}$ : $G \times X_{r} \rightarrow X_{r}$ for each $r \in R$.

Theorem 5.5. The family $\left(X_{R}, \varpi_{R}\right)$ defined above is the versal family of tangential deformations of $(X, \varpi)$.

Proof. Let $\kappa: T_{0} R \rightarrow h^{1}(X, T X)$ denote the Kodaira-Spencer map associated to the family $\left(X_{R}, \varpi_{R}\right)$ at 0 . Let $\left(C=\mathrm{id}+C^{\prime}, \theta\right)$ be a given element in $\Xi$ close to (id, 0$)$. Then $\left(C^{\prime}, \theta\right)$ is an element of $T_{0} R=$ End $\mathbb{C}^{s} \oplus H^{0}\left(X, \Omega_{X}^{1}\right)^{s}$, close to zero, that can be seen as a cocycle in $C_{h}^{1}\left(\mathbb{C}^{s}, \Omega^{0,0}(X, T \mathcal{F})\right) \oplus C_{h}^{0}\left(\mathbb{C}^{s}, \Omega^{0,1}(X, T \mathcal{F})\right)$. An easy computation shows that $\kappa\left(C^{\prime}, \theta\right)$ is just the cohomology class in $H_{\mathbb{C}^{s}}^{1}(X, T \mathcal{F})$ of that cocycle. This implies that: (i) the restriction of $\kappa$ to End $\mathbb{C}^{s}$ is just the map $\tau$ in (9), and (ii) the restriction of $\kappa$ to $T_{0} R_{0} \equiv H^{0}\left(X, \Omega_{X}^{1}\right)^{s}$ is a section of the morphism $\chi$ in (9). Hence $\kappa$ is an isomorphism and the statement follows from Proposition 4.4.

Let $\mathcal{K}_{X}$ denote the Kuranishi space of $X$, that is, the parameter space of the versal family of deformations of the complex manifold $X$. Recall that $\mathcal{K}_{X}$ is a (possibly singular) analytic space whose tangent space at the distinguished point $0 \in \mathcal{K}_{X}$ is naturally identified 
to $H^{1}\left(X, \Theta_{X}\right)$. The restriction $X_{R_{0}}$ to $R_{0}$ of the family $X_{R}$ can be seen as a family of deformations of the complex structure of $X$. Hence there is a well defined forgetful map of germs of analytic spaces

$$
\phi:\left(R_{0}, 0\right) \rightarrow\left(\mathcal{K}_{X}, 0\right)
$$

Moreover its tangent map $d_{0} \phi$ at 0 coincides with the morphism

$$
H^{1}(X, T \mathcal{F}) \rightarrow H^{1}\left(X, \Theta_{X}\right)
$$

induced by the decomposition $T X=T \mathcal{F} \oplus T \mathcal{G}$. More precisely, given an element $\theta=$ $\left(\theta^{1}, \ldots, \theta^{s}\right)$ in $T_{0} R_{0}$ with $\theta^{i}=\sum a_{j}^{i} \alpha^{j}+\sum b_{k}^{i} \beta^{k}$, its image $d_{0} \phi(\theta)$ is the vector valued $(0,1)$-form

$$
\tilde{\theta}=\sum \overline{\theta^{i}} \otimes v_{i}
$$

Since holomorphic forms are closed and nonexact, one can see using local coordinates fufilling (4) that $\tilde{\theta}$ defines a cohomology class in $H^{1}\left(X, \Theta_{X}\right)$ which is not trivial unless $\theta^{i}=0$ for all $i=1, \ldots, s$. This implies that $\phi$ is an embedding, proving the following statement.

Theorem 5.6. Let $X$ be a compact manifold in the class $\mathcal{C}$ such that $s=\operatorname{dim}_{\mathbb{C}} T_{X}=$ $\operatorname{dim}_{\mathbb{C}} \mathfrak{h}_{X} / \mathfrak{h}_{X}^{1} \neq 0$. Then the Kuranishi space $\mathcal{K}_{X}$ of $X$ contains a smooth subspace of dimension $s \cdot b_{1}(X) / 2$. More precisely, the canonical morphism $H^{1}(X, T \mathcal{F}) \equiv$ $H^{1}\left(X, \mathcal{O}_{X}\right)^{s} \rightarrow H^{1}\left(X, \Theta_{X}\right)$ is injective and its image are unobstructed infinitesimal deformations of the complex structure of $X$.

We shall distinguish two particular types of tangential deformations defined as follows:

Definition 5.7. Let $r=(C, \theta) \in R$ be given and set $\theta=\left(\theta^{1}, \ldots, \theta^{s}\right)$. We say that the associated tangential deformation $\left(X_{r}, \varpi_{r}\right)$ is

(i) a purely tangential deformation, or $t$-deformation, of $(X, \varpi)$ if $\theta^{i} \in\left\langle\alpha^{1}, \ldots, \alpha^{s}\right\rangle$, i.e. the coefficients $b_{k}^{i}$ in (10) are all zero,

(ii) a basic deformation, or b-deformation, of $(X, \varpi)$ if $\theta^{i} \in\left\langle\beta^{1}, \ldots, \beta^{p-s}\right\rangle$, i.e. the coefficients $a_{j}^{i}$ in (10) are all zero.

Remarks 5.8. (a) The holomorphic and invariant foliation $\mathcal{G}$, which is transversal and complementary to $\mathcal{F}$, remains holomorphic and invariant for each $t$-deformation of $(X, \varpi)$. On the other hand the foliation $\mathcal{F}$ remains holomorphic for each $b$-deformation of $(X, \varpi)$.

(b) If $\left(X_{r}, \varpi_{r}\right)$ is a $t$-deformation (resp. $b$-deformation) of $(X, \varpi)$ then $(X, \varpi)$ is a $t$-deformation (resp. $b$-deformation) of $\left(X_{r}, \varpi_{r}\right)$.

Note also that each small tangential deformation $\left(X_{r^{\prime}}, \varpi_{r^{\prime}}\right)$ of $(X, \varpi)$ can be seen as a $b$-deformation of a $t$-deformation $\left(X_{r}, \varpi_{r}\right)$ of $(X, \varpi)$. More precisely, if $X_{r^{\prime}}$ is determined by 1 -forms $\tilde{\alpha}^{i}=\alpha^{i}+\sum \overline{a_{j}^{i}} \overline{\alpha^{j}}+\sum \overline{b_{k}^{i}} \overline{\beta^{k}}$ then the manifold $X_{r}$ determined by the 1 -forms $\hat{\alpha}^{i}=\alpha^{i}+\sum \overline{a_{j}^{i}} \overline{\alpha^{j}}$ is a $t$-deformation of $X$. Moreover, since $\tilde{\alpha}^{i}=\hat{\alpha}^{i}+\sum \overline{b_{k}^{i}} \overline{\beta^{k}}$, the manifold $X_{r^{\prime}}$ is a $b$-deformation of $X_{r}$.

Notice that, as $X$ is a tangential deformation of $X_{r}$, the previous argument also proves that $\left(X_{r^{\prime}}, \varpi_{r^{\prime}}\right)$ can be seen as a $t$-deformation of a $b$-deformation of $(X, \varpi)$. 
As remarked in the proof of Proposition 5.2, local holomorphic functions on $X$ that are basic with respect to $\mathcal{F}$ are also holomorphic on $X_{r}$ and basic with respect to $\mathcal{F}_{r}$. By definition, the global 1-forms $\alpha_{r}^{i}$ are of type $(1,0)$ on $X_{r}$ and they are also closed by construction. Hence $\alpha_{r}^{i}$ are holomorphic forms on $X_{r}$. It follows that, for each $r \in R$, one has $h^{1,0}\left(X_{r}\right)=h^{1,0}(X), h^{0,1}\left(X_{r}\right)=h^{0,1}(X)$ and $b_{1}(X)=h^{1,0}\left(X_{r}\right)+h^{0,1}\left(X_{r}\right)$. In particular, the space of holomorphic 1-forms on $X_{r}$ can be decomposed as

$$
H^{0}\left(X_{r}, \Omega_{X_{r}}^{1}\right)=\left\langle\alpha_{r}^{1}, \ldots, \alpha_{r}^{s}\right\rangle \oplus\left\langle\beta^{1}, \ldots, \beta^{p-s}\right\rangle .
$$

Proposition 5.9. Let $\mathfrak{a}_{X}$ be an Abelian algebra satisfying $\mathfrak{h}_{X}=\mathfrak{a}_{X} \oplus \mathfrak{h}_{X}^{1}$ and let $X_{r}$ be a tangential deformation of $X$.

(i) If $\mathfrak{a}_{X}$ is in the center of $\mathfrak{h}_{X}$ then $\mathfrak{h}_{X_{r}}^{1}=\mathfrak{h}_{X}^{1}$.

(ii) If $X_{r}$ is a b-deformation of $X$ then $\mathfrak{a}_{X} \subset \mathfrak{h}_{X_{r}}$ and $\mathfrak{h}_{X_{r}}=\mathfrak{a}_{X} \oplus \mathfrak{h}_{X_{r}}^{1}$.

If both conditions are fulfilled then $\mathfrak{h}_{X_{r}}=\mathfrak{h}_{X}$.

Proof. (i) Let $w \in \mathfrak{h}_{X}^{1}$ be given. Notice that $\alpha^{i}(w)=\alpha_{r}^{i}(w)=0$ for $i=1, \ldots, s$. Since $\left[\mathfrak{a}_{X}, \mathfrak{h}_{X}^{1}\right]=0, w$ is written locally as

$$
w=\sum_{k=1}^{n-s} f^{k}(z) \frac{\partial}{\partial z^{k}},
$$

where all $f^{k}$ are holomorphic functions of $z^{1}, \ldots, z^{n-s}$. It follows that $w$ is of type $(1,0)$ on $X_{r}$. Now the same argument used in the proof of Proposition 5.2 shows that $w$ is in fact holomorphic in $X_{r}$. The original manifold $X$ is a tangential deformation of $X_{r}$ (cf. Remark 5.4), so $\mathfrak{h}_{X_{r}}^{1}$ does not contain any additional tangent fields besides those arising in $X$.

(ii) If $X_{r}$ is a $b$-deformation of $X$ then the elements $v \in \mathfrak{a}_{X}$ are still holomorphic on $X_{r}$, in view of the identities (11) to (14).

We now discuss the tangential deformations of the examples of Kähler manifolds introduced in Section 2.

Example 5.10 (Deformations of tori). If $X$ is a complex torus $\mathbb{T}^{s}=\mathbb{C}^{s} / \Lambda$ then $H^{0}\left(\mathbb{T}^{s}, \Omega_{\mathbb{T}^{s}}^{1}\right)^{s}$ can be identified with the dual space of $H^{1}\left(\mathbb{T}^{s}, \Theta_{\mathbb{T}^{s}}\right)$. In that case the space $R$ is just the product of $\mathrm{GL}(s, \mathbb{C})$ with the versal space of deformations of the complex manifold $\mathbb{T}^{s}$, as described by Kodaira and Spencer [23].

Example 5.11 (Deformations of principal torus bundles). Assume that $X$ is the total space of a principal torus bundle $\mathbb{T}^{s} \rightarrow X \rightarrow B$ where $\mathbb{T}^{s}=\mathbb{C}^{s} / \Lambda$. As already noticed (cf. Example 2.3), when $X$ is Kähler then $B$ is also a Kähler manifold and the bundle is flat. The possible choices of the $s$-tuple $\left(\alpha^{1}, \ldots, \alpha^{s}\right)$ correspond exactly to the different flat connections on that bundle.

In this situation the space of basic deformations of the action is naturally identified to $H^{0}\left(B, \Omega_{B}^{1}\right)^{s} \cong H^{1}\left(B, \mathcal{O}_{B}^{s}\right)$. The exact sequence of sheaves $0 \rightarrow \Lambda \rightarrow \mathcal{O}_{B}^{s} \rightarrow$ $\mathcal{O}_{B}^{s} / \Lambda \rightarrow 0$ over $B$ induces the exact cohomology sequence

$$
\cdots \rightarrow H^{1}(B, \Lambda) \rightarrow H^{1}\left(B, \mathcal{O}_{B}^{s}\right) \rightarrow H^{1}\left(B, \mathcal{O}_{B}^{s} / \Lambda\right) \stackrel{\tau}{\rightarrow} H^{2}(B, \Lambda) \rightarrow \cdots
$$


It follows that $H^{1}\left(B, \mathcal{O}_{B}^{s}\right)$ is mapped onto $\operatorname{ker} \tau$, which is the space of (isomorphism classes of) topologically trivial $\mathbb{C}^{s} / \Lambda$-principal bundles over $B$. Replacing $B$ by a finite (unramified) covering we can suppose that $H^{2}(B, \Lambda)$ has no torsion. In that case each topologically trivial principal torus bundle over $B$ can be obtained from $X$ by a suitable basic deformation of the action and, in particular, the trivial bundle is a tangential deformation of $(X, \varpi)$.

In general, each tangential deformation of $(X, \varpi)$ is a principal torus bundle over $B$. Purely tangential deformations $r \in R$ just deform the fiber $\mathbb{C}^{s} / \Lambda_{r}$.

Example 5.12 (Deformations of suspensions). Assume that $X$ is a suspension over a torus $\mathbb{T}^{s}=\mathbb{C}^{s} / \Lambda$, that is, $X=F \times_{\Lambda} \mathbb{C}^{s}$. It follows from Remark 5.8(a) that each purely tangential deformation of $X$ is still a suspension, although this is not true for general tangential deformations of $X$.

If $X$ is a suspension one has $H^{0}\left(X, \Omega_{X / \mathcal{F}}^{1}\right)=H^{0}\left(F, \Omega_{F}^{1}\right)$. Therefore if $b_{1}(F)=0$, or, what is equivalent, $b_{1}(X)=2 s$, then $X$ has no basic deformations and each tangential deformation of $X$ is again a suspension.

We end this section stating several general properties of tangential deformations of compact manifolds of the class $\mathcal{C}$ endowed with a locally free $\mathbb{C}^{s}$-action.

If $\tilde{X} \rightarrow X$ is an unramified covering then $\tilde{X}$ is also endowed with a locally free $\mathbb{C}^{s}$ action in a natural way. Notice however that the maximal rank of such an action on $\tilde{X}$ can increase, as $\operatorname{dim}_{\mathbb{C}} \mathfrak{h}_{\tilde{X}} / \mathfrak{h}_{\tilde{X}}^{1} \geq s$. The following statement follows straightforward from the definitions.

Proposition 5.13. A finite covering $X_{r}^{\prime}$ of a tangential deformation $X_{r}$ of $X$ is a tangential deformation of a finite covering $X^{\prime}$ of $X$. Conversely, a tangential deformation $X_{r}^{\prime}$ of a finite covering $X^{\prime}$ of $X$ is a finite covering of a tangential deformation $X_{r}$ of $X$.

Proposition 5.14. Let $X$ be a geometrically ruled manifold in the class $\mathcal{C}$. Then $X_{r}$ is a geometrically ruled compact complex manifold for each $r \in R$.

Proof. Let $X$ be a compact manifold in the class $\mathcal{C}$ endowed with a locally free $\mathbb{C}^{s}$ action $\varpi$, and assume that $X$ is the total space of a locally trivial fiber bundle, $\varphi: X \rightarrow B$, with fiber $\mathbb{C} P^{k}$ and defined by a cocyle $\left\{g_{i j}\right\}$ in $H^{1}\left(B, \operatorname{PGL}\left(k+1, \mathcal{O}_{B}\right)\right)$. Then $B$ is also in the class $\mathcal{C}$. Since automorphisms of $X$ close to the identity must preserve the fibration (cf. [5]), each fundamental vector field $v$ of the action $\varpi$ projects to a nonvanishing vector field $\hat{v}$ on $B$. These vector fields define a locally free $\mathbb{C}^{s}$-action $\hat{\omega}$ on $B$ and the projection $\varphi$ is equivariant with respect to the $\mathbb{C}^{S}$-actions. Notice that every global holomorphic 1-form on $X$ vanishes on the fibers of $\varphi$. Hence $\varphi^{*}: H^{0}\left(B, \Omega_{B}^{1}\right) \rightarrow H^{0}\left(X, \Omega_{X}^{1}\right)$ is an isomorphism. Using this identification, one can associate, to each tangential deformation $X_{r}$ given by a pair $r=(C, \theta)$, a tangential deformation $B_{r}$ defined by the same expression (10). Then the projection $\varphi: X_{r} \rightarrow B_{r}$ is holomorphic.

Now, as the $\mathbb{C}^{S}$-action $\varpi$ is transverse and preserves the fibration, the cocycle $\left\{g_{i j}\right\}$ can be chosen to be constant along the orbits of the $\mathbb{C}^{S}$-action $\hat{\omega}$ on $B$. This implies that the matrix-valued functions $g_{i j}$ are still holomorphic on $B_{r}$ and therefore they define $X_{r}$ as a geometrically ruled manifold over $B_{r}$. 
Proposition 5.15. A tangential deformation $X_{r}$ of $X$ uniquely determines a tangential deformation $\operatorname{Alb}(X)_{r}$ of the Albanese torus $\operatorname{Alb}(X)$ of $X$ such that $\operatorname{Alb}(X)_{r}=\operatorname{Alb}\left(X_{r}\right)$, i.e. $\mathrm{Alb}(X)_{r}$ is the Albanese torus of $X_{r}$.

Proof. The locally free $\mathbb{C}^{s}$-action $\varpi$ on $X$ induces a locally free $\mathbb{C}^{s}$-action $\hat{\omega}$ on the corresponding Albanese torus $\operatorname{Alb}(X)$ and the natural map $\phi: X \rightarrow \operatorname{Alb}(X)$ is equivariant with respect to these actions. Since the map $\phi$ induces an isomorphism $\phi^{*}: H^{0}\left(\operatorname{Alb}(X), \Omega_{\operatorname{Alb}(X)}^{1}\right) \rightarrow H^{0}\left(X, \Omega_{X}^{1}\right)$, there is a natural correspondence, as the one considered in the proof of the above proposition, associating to each tangential deformation $X_{r}$ of $X$ a tangential deformation $\operatorname{Alb}(X)_{r}$ of $\operatorname{Alb}(X)$. It follows from the definitions that $\operatorname{Alb}(X)_{r}=\operatorname{Alb}\left(X_{r}\right)$.

Proposition 5.16. The manifolds $X$ and $X_{r}$ have the same plurigenera for all $r \in R$. In particular the Kodaira dimension $\operatorname{kod}\left(X_{r}\right)$ of $X_{r}$ is equal to $\operatorname{kod}(X)$ for each $r \in R$.

Proof. Let $\left(t^{1}, \ldots, t^{s}, z^{1}, \ldots, z^{n-s}\right)$ be local coordinates of $X$ satisfying (4) and let $\alpha_{r}^{i}$ be the global holomorphic 1 -forms on $X$ defined by (10). Notice that $z^{j}$ are also holomorphic functions on $X_{r}$. A local section of $K_{X_{r}}^{\otimes m}$ is of the form $f(t, z) \eta^{m}$ where $\eta=\alpha^{1} \wedge \cdots \wedge$ $\alpha^{s} \wedge d z_{i}^{1} \wedge \cdots \wedge d z_{i}^{n-s}$ and $f=f(t, z)$ is a holomorphic function.

Nakamura and Ueno have proved that, for each compact complex manifold $Y$, the group $\operatorname{Aut}_{\mathbb{C}}^{0}(Y)$ acts trivially on $H^{0}\left(Y, K_{Y}^{m}\right)$ (cf. [32, Corollary 14.8]). Applying this result to the $\mathbb{C}^{s}$-action we see that $f$ is necessarily a basic function, i.e. $f=f(z)$ only depends on the coordinates $\left(z^{1}, \ldots, z^{n-s}\right)$ transverse to $\mathcal{F}$. Therefore $H^{0}\left(X_{r}, K_{X_{r}}^{\otimes m}\right)$ is naturally identified to the space of basic global sections of the line bundle $\left(\operatorname{det} v^{*} \mathcal{F}_{r}\right)^{m}$ and this space is independent of $r \in R$.

If $X$ is a Kähler manifold then Kodaira's stability theorem implies that $X_{r}$ is also Kähler for every $r$ in a neighborhood of (id, 0) in $R$, and we will prove the following result, stating that the class of Kähler manifolds is stable under tangential deformations. In sharp contrast the class $\mathcal{C}$ is not stable under small deformations. We do not know if tangential deformations of Fujiki manifolds still belong to the class $\mathcal{C}$.

Theorem 5.17. Assume that $X$ is a Kähler manifold. Then $X_{r}$ is also a Kähler manifold for each $r \in R$.

We postpone the proof of this theorem to Section 7, where the assertion will be a corollary of general results on the structure of such manifolds. Granting the theorem, we can state the following two propositions:

Proposition 5.18. Assume that $X$ and $X_{r}$ belong to the class $\mathcal{C}$. Then

(i) $h^{k, 0}(X)=h^{k, 0}\left(X_{r}\right)$ and $h^{0, k}(X)=h^{0, k}\left(X_{r}\right)$ for each $k$,

(ii) $h^{1,1}(X)=h^{1,1}\left(X_{r}\right)$.

If $X$ is a Kähler manifold, the above identities hold for each $r \in R$. 
Proof. Lemma 2.10 implies that there is a well defined $\mathbb{C}$-linear map from $H^{0}\left(X, \Omega_{X}^{k}\right)$ to $H^{0}\left(X_{r}, \Omega_{X_{r}}^{k}\right)$ determined by the condition

$$
\gamma=\sum_{0 \leq|I| \leq \ell} \alpha^{I} \wedge \gamma^{I} \mapsto \gamma_{r}=\sum_{0 \leq|I| \leq \ell} \alpha_{r}^{I} \wedge \gamma^{I},
$$

where $\ell=\min (k, s), \alpha_{r}^{I}=\alpha_{r}^{i_{1}} \wedge \cdots \wedge \alpha_{r}^{i_{|I|}}, \alpha_{r}^{i}=\alpha^{i}+\overline{\theta^{i}}$ and $\gamma^{I}$ are global basic forms. Clearly this map is in fact an isomorphism. Preservation of $b_{2}, h^{2,0}, h^{0,2}$ implies that of $h^{1,1}$.

Proposition 5.19. Assume that $X$ is a Kähler manifold. Then the family $\left(X_{R}, \varpi_{R}\right)$ is the versal family of $\left(X_{r}, \varpi_{r}\right)$ at each point $r \in R$.

Proof. In that case $X_{r}$ is a Kähler manifold for each $r \in R$ and $H^{1}\left(X_{r}, \mathcal{O}_{X_{r}}\right)$ is isomorphic to $H^{0}\left(X_{r}, \Omega_{X_{r}}^{1}\right)$. Moreover, the dimension of these vector spaces does not vary with $r \in R$. Hence the argument used to prove Theorem 5.5 also applies to each $X_{r}$ with $r \in R$.

\section{The approximation theorem}

In this section we prove a key result for describing the structure of compact complex manifolds $X$ in the class $\mathcal{C}$ endowed with a locally free holomorphic $\mathbb{C}^{s}$-action. Namely, we prove that such a manifold can be approximated by tangential deformations $X_{\epsilon}$ of $X$ that are suspensions over a torus of dimension $s$, i.e. $X_{\epsilon}$ belonging to the class of manifolds described in Example 2.4.

We shall make use of the following criterion, which is valid for arbitrary compact complex manifolds.

Lemma 6.1. Let $X$ be a compact complex manifold endowed with a locally free action of $\mathbb{C}^{s}$ and let $v_{1}, \ldots, v_{s}$ be a basis of fundamental vector fields of the action. Then $X$ is a suspension over a torus $T=\mathbb{C}^{s} / \Lambda$, i.e. $X=F \times{ }_{\Lambda} \mathbb{C}^{s}$ for a suitable representation $\Lambda \rightarrow \operatorname{Aut}_{\mathbb{C}}(F)$, if and only if one can choose holomorphic 1-forms $\alpha^{j}$ on $X$ satisfying $\alpha^{j}\left(v_{i}\right)=\delta_{i}^{j}$ and such that the set of periods of $\alpha^{1}, \ldots, \alpha^{s}$,

$$
\Lambda=\left\{\left(\int_{\gamma} \alpha^{1}, \ldots, \int_{\gamma} \alpha^{s}\right) \mid \gamma \in H_{1}(X, \mathbb{Z})\right\}
$$

is a lattice of $\mathbb{C}^{s}$.

Proof. For each $i=1, \ldots, s$ let $f^{i}$ be a multivalued holomorphic function such that $\alpha^{i}=d f^{i}$. If the set of periods $\Lambda$ is a lattice of $\mathbb{C}^{s}$ then $f=\left(f^{1}, \ldots, f^{s}\right)$ is a well defined holomorphic submersion from $X$ onto $\mathbb{C}^{s} / \Lambda$. The converse is clear. 
Remark 6.2. The fiber space $F$ in the above theorem can always be taken connected. Indeed, if the fibers of the submersion $f: X \rightarrow T$ are not connected, one can consider the Stein factorization of $f$,

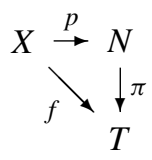

Since the map $p$ is finite and $f$ is a submersion, the map $\pi$ is necessarily an unbranched cover, thus $N$ is also a torus that covers $T$. The map $p$ is then the desired suspension.

The following result is an improvement of Theorem 2.16 in [29]. For our purposes here, the essential point of the theorem is that the manifold $X_{\epsilon}$, which approximates $X$ and is a suspension over a torus, can be taken to be a basic deformation of $X$.

Theorem 6.3. Let $X$ be a compact complex manifold in the class $\mathcal{C}$ endowed with a locally free $\mathbb{C}^{s}$-action. There is an arbitrarily small b-deformation $X_{\epsilon}$ of $X$ which is a suspension over a complex torus $T=\mathbb{C}^{S} / \Lambda$. More precisely, there is a connected compact complex manifold $F$ and a group representation $\rho: \pi_{1}(T)=\Lambda \rightarrow \operatorname{Aut}_{\mathbb{C}}(F)$ such that $X_{\epsilon}$ is the suspension of $\rho$.

Proof. First, we claim that there exist arbitrarily small holomorphic 1-forms $\theta^{i}, \eta^{i}$ on $X$ such that, if we set $\tilde{\alpha}^{i}=\alpha^{i}+\overline{\theta^{i}}+\eta^{i}$, then

$$
\Lambda=\left\{\left(\int_{\gamma} \tilde{\alpha}^{1}, \ldots, \int_{\gamma} \tilde{\alpha}^{s}\right) \mid \gamma \in H_{1}(X, \mathbb{Z})\right\}
$$

is a lattice of $\mathbb{C}^{s}$. The proof is essentially the same as the one given in [29, p. 490]. Let $\left\{\alpha^{1}, \ldots, \alpha^{s}, \beta^{1}, \ldots, \beta^{p-s}\right\}$ be the basis of $H^{0}\left(X, \Omega_{X}^{1}\right)$ considered in (5). We decompose

$$
\theta^{i}=\theta_{t}^{i}+\theta_{b}^{i}=\sum a_{j}^{i} \alpha^{j}+\sum b_{k}^{i} \beta^{k}, \quad \eta^{i}=\eta_{t}^{i}+\eta_{b}^{i}=\sum c_{j}^{i} \alpha^{j}+\sum d_{k}^{i} \beta^{k}
$$

It follows from Remark 5.3(c) that the 1-forms $\alpha^{i}+\overline{\theta^{i}}+\eta_{t}^{i}$ determine a complex manifold endowed with a $\mathbb{C}^{s}$-action which is isomorphic to a suitable $\left(X_{r^{\prime}}, \varpi_{r^{\prime}}\right)$ close to $(X, \varpi)$. Since $\tilde{\alpha}^{i}$ are holomorphic 1 -forms on $X_{r^{\prime}}$, Lemma 6.1 implies that the manifold $X_{r^{\prime}}$ is a suspension over a torus.

As noticed in Remark 5.8(b), we can view $\left(X_{r^{\prime}}, \varpi_{r^{\prime}}\right)$ as a $t$-deformation of a suitable $\left(X_{r}, \varpi_{r}\right)$ close to $(X, \varpi)$ with the property that $\left(X_{r}, \varpi_{r}\right)$ is a $b$-deformation of $(X, \varpi)$. But $X_{r}$ is a suspension over a torus as $t$-deformations of suspensions are again suspensions (cf. Example 5.12). Hence $\left(X_{r}, \varpi_{r}\right)$ fulfills the required conditions, as Remark 6.2 ensures that the fiber $F$ of the suspension can always be taken connected.

It follows from the above theorem that there is an exact sequence of groups $1 \rightarrow \pi_{1}(F)$ $\rightarrow \pi_{1}(X) \rightarrow \mathbb{Z}^{2 s} \rightarrow 1$ 
Remarks 6.4. (a) It follows from Remark 5.3(a) that $X_{\epsilon}$ is real-analytically isomorphic to $X$. More precisely, as the two complex manifolds $X$ and $X_{\epsilon}$ have the same underlying real-analytic structure, the identity map id: $X \rightarrow X_{\epsilon}$ is real-analytic.

(b) Assume that $X$ is a suspension over a torus $T=\mathbb{C}^{s} / \Lambda$, i.e. $X=F \times \Lambda \mathbb{C}^{s}$. If $T^{\prime}$ is a torus isogenous to $T$ then $T^{\prime}=\mathbb{C}^{s} / \Lambda^{\prime}$, where $\Lambda^{\prime}$ is a sublattice of $\Lambda$, and there is an Abelian finite covering $X^{\prime}$ of $X$ which is a suspension over $T^{\prime}$. From this fact one deduces easily that if $X$ is a suspension over an $s$-dimensional torus, where $s=$ $\operatorname{dim}_{\mathbb{C}} T_{X}=\operatorname{dim}_{\mathbb{C}} \mathfrak{h}_{X} / \mathfrak{h}_{X}^{1}$, then $X$ has an Abelian finite covering $X^{\prime}$ that is a suspension over $T_{X}=\Phi\left(\operatorname{Aut}_{\mathbb{C}}^{0}(X)\right) \subset \operatorname{Alb}(X)$.

(c) Let $X$ be a Kähler manifold satisfying the hypothesis of the above theorem. Then $X_{\epsilon}$ is the suspension $F \times{ }_{\Lambda} \mathbb{C}^{s}$ of a representation $\rho: \Lambda \rightarrow \operatorname{Aut}_{\mathbb{C}}(F)$. Since $X_{\epsilon}$ is a Kähler manifold, there is a sublattice $\Lambda^{\prime}$ of $\Lambda$ such that $\rho\left(\Lambda^{\prime}\right) \subset \operatorname{Aut}_{\mathbb{C}}^{0}(F)$ (cf. 2.4). Then the suspension $X_{\epsilon}^{\prime}=F \times{ }_{\Lambda^{\prime}} \mathbb{C}^{s}$ is an Abelian finite covering of $X_{\epsilon}$. Notice that $X_{\epsilon}^{\prime}$ is topologically isomorphic to the product $F \times T^{\prime}$, where $T^{\prime}=\mathbb{C}^{s} / \Lambda^{\prime}$, and that $X_{\epsilon}^{\prime}$ can be seen as a $b$-deformation of a finite covering $X^{\prime}$ of $X$ (cf. 5.13 and 5.4).

(d) Any (unramified) finite covering $X^{\prime}$ of $X$ is naturally endowed with a $\mathbb{C}^{s}$-action but the maximal rank can increase, that is

$$
\operatorname{dim}_{\mathbb{C}} \mathfrak{h}_{X^{\prime}} / \mathfrak{h}_{X^{\prime}}^{1} \geq \operatorname{dim}_{\mathbb{C}} \mathfrak{h}_{X} / \mathfrak{h}_{X}^{1}
$$

Examples where this inequality is strict can be constructed by considering suspensions of bielliptic surfaces.

A more precise description of the fiber manifold $F$ and of the representation $\rho$ : $\Lambda \rightarrow \operatorname{Aut}_{\mathbb{C}}(F)$ in the above theorem can be obtained by considering (unramified) finite coverings $X^{\prime}$ of the manifold $X$. This is the content of the following proposition. It can be seen as an improvement of the approximation theorem and will play a key role in the structure theorems of the next section.

Proposition 6.5. Let $X$ be a compact Kähler manifold such that $s=\operatorname{dim}_{\mathbb{C}} T_{X}=$ $\operatorname{dim}_{\mathbb{C}} \mathfrak{h}_{X} / \mathfrak{h}_{X}^{1}>0$. There is a finite Abelian covering $X_{\epsilon}^{\prime}$ of an arbitrarily small $b$ deformation $X_{\epsilon}$ of $X$, which is the suspension over a torus $T=\mathbb{C}^{s^{\prime}} / \Lambda$, of dimension $s^{\prime} \geq s$, associated to a representation $\rho: \Lambda \rightarrow \operatorname{Aut}_{\mathbb{C}}(F)$ with the following properties:

(i) $F$ is a connected compact Kähler manifold with $\mathfrak{h}_{F} / \mathfrak{h}_{F}^{1}=0$, i.e. $F$ has no nonsingular holomorphic vector fields,

(ii) $\rho(\Lambda) \subset \operatorname{Aut}_{\mathbb{C}}^{0}(F)$.

Remark 6.6. The manifold $X_{\epsilon}^{\prime}$ can also be seen as a (small) $b$-deformation of a finite Abelian covering $X^{\prime}$ of $X$. This follows from Proposition 5.13.

Proof of Proposition 6.5. Let $\mathfrak{a}_{X}$ be a central Abelian subalgebra of $\mathfrak{h}_{X}$ with $\mathfrak{h}_{X}=$ $\mathfrak{a}_{X} \oplus \mathfrak{h}_{X}^{1}$. It defines a locally free $\mathbb{C}^{S}$-action on $X$, and by Theorem 6.3 there is an arbitrarily small $b$-deformation $X_{\epsilon}$ of $X$ which is the suspension $F_{1} \times{ }_{\Lambda_{1}} \mathbb{C}^{s}$ associated to a representation $\rho_{1}: \Lambda_{1} \rightarrow \operatorname{Aut}_{\mathbb{C}}\left(F_{1}\right)$. Notice that, applying Proposition 5.9, we have $\mathfrak{a}_{X} \subset \mathfrak{h}_{X_{\epsilon}}$. Therefore we can choose $\mathfrak{a}_{X_{\epsilon}}=\mathfrak{a}_{X}$ as the complement of $\mathfrak{h}_{X_{\epsilon}}^{1}$ in $\mathfrak{h}_{X_{\epsilon}}$. 
As in Remark 6.4(c) above, we consider the sublattice $\Lambda_{1}^{\prime}=\rho_{1}^{-1}\left(\operatorname{Aut}_{\mathbb{C}}^{0}\left(F_{1}\right)\right)$ of $\Lambda_{1}$. Then the suspension $X_{\epsilon}^{1}=F_{1} \times_{\Lambda_{1}^{\prime}} \mathbb{C}^{s}$ is an Abelian finite covering of $X_{\epsilon}=X_{\epsilon}^{1} / \Gamma_{1}$ where $\Gamma_{1}=\Lambda_{1} / \Lambda_{1}^{\prime}$. More precisely, each vector field on $X_{\epsilon}$ lifts to a unique vector field on $X_{\epsilon}^{1}$ and therefore we can identify $\mathfrak{a}_{X_{\epsilon}}=\mathfrak{a}_{X}$ with an Abelian subalgebra of $\mathfrak{h}_{X_{\epsilon}^{1}}$ that we still denote by $\mathfrak{a}_{X}$. Then there are $v_{i} \in \mathfrak{a}_{X}$ such that the monodromies $\rho_{1}(\gamma)$ for the set of elements $\gamma \in \Lambda_{1}$ generating $\Gamma_{1}$ lie in $\left\langle\gamma_{1}, \ldots, \gamma_{k}\right\rangle$, where $\gamma_{i}=\exp _{1}\left(v_{i}\right)$.

If $F_{1}$ has no vector fields without zeros we are done. So, assume that $\mathfrak{h}_{F_{1}} / \mathfrak{h}_{F_{1}}^{1} \neq 0$. In that case we choose a subalgebra $\mathfrak{a}_{F_{1}}$ of the center of $\mathfrak{h}_{F_{1}}$ such that $\mathfrak{h}_{F_{1}}=\mathfrak{a}_{F_{1}} \oplus \mathfrak{h}_{F_{1}}^{1}$ (cf. Proposition 1.6). Since $\rho_{1}\left(\Lambda_{1}^{\prime}\right)$ is contained in $\operatorname{Aut}_{\mathbb{C}}^{0}\left(F_{1}\right)$, each element $\hat{w}$ of $\mathfrak{a}_{F_{1}}$ is $\rho_{1}\left(\Lambda_{1}^{\prime}\right)$-invariant. This implies that $\hat{w}$ is the restriction of a globally defined holomorphic vector field $w$ on $X_{\epsilon}^{1}$ without zeros and commuting with $\mathfrak{a}_{X}$. More precisely, $\hat{w}$ is the projection over $F_{1} \times \Lambda_{1} \mathbb{C}^{s}$ of the vector field $(w, 0)$ on the product manifold $F_{1} \times \mathbb{C}^{s}$. By construction, $\mathfrak{a}_{X_{\epsilon}^{1}}=\mathfrak{a}_{X} \oplus \mathfrak{a}_{F_{1}}$ is Abelian and $\mathfrak{h}_{X_{\epsilon}^{1}}=\mathfrak{a}_{X_{\epsilon}^{1}} \oplus \mathfrak{h}_{X_{\epsilon}^{1}}^{1}$. Denote $r=\operatorname{dim} \mathfrak{a}_{X_{\epsilon}^{1}}>s$, and repeat the above construction beginning with $X_{\epsilon}^{1}$ instead of $X$.

There is a small $b$-deformation $X_{\delta}^{1}$ of $X_{\epsilon}^{1}$ which is the suspension $F_{2} \times_{\Lambda_{2}} \mathbb{C}^{r}$ over a torus $\mathbb{C}^{r} / \Lambda_{2}$, associated to a representation $\rho_{2}: \Lambda_{2} \rightarrow \operatorname{Aut}_{\mathbb{C}}\left(F_{2}\right)$. We see, using Proposition 5.13, that $X_{\delta}^{1}$ is a finite Abelian covering of a $b$-deformation $X_{\delta}$ of $X$. And, as above, $\mathfrak{a}_{X_{\epsilon}^{1}}$ is an Abelian subalgebra of $\mathfrak{h}_{X_{\delta}^{1}}$ with $\mathfrak{h}_{X_{\delta}^{1}}=\mathfrak{a}_{X_{\epsilon}^{1}} \oplus \mathfrak{h}_{X_{\delta}^{1}}^{1}$. In particular, $\gamma_{i}=\exp _{1}\left(v_{i}\right)$ can be seen as holomorphic transformations of $X_{\delta}^{1}$.

We set $\Lambda_{2}^{\prime}=\rho_{2}^{-1}\left(\operatorname{Aut}_{\mathbb{C}}^{0}\left(F_{2}\right)\right)$ and we define $X_{\delta}^{2}=F_{2} \times_{\Lambda_{2}^{\prime}} \mathbb{C}^{r}$. Then $X_{\delta}^{2}$ is a finite Abelian covering of $X_{\delta}^{1}=X_{\delta}^{2} / \Gamma_{2}$ where $\Gamma_{2}=\Lambda_{2} / \Lambda_{2}^{\prime}$. Also as above, $\mathfrak{a}_{X_{\epsilon}^{1}}=\mathfrak{a}_{X} \oplus \mathfrak{a}_{F_{1}}$ is naturally included in $\mathfrak{h}_{X_{\delta}^{2}}$. It follows in particular that the automorphisms $\gamma_{i}=\exp _{1}\left(v_{i}\right)$ for $v_{i} \in \mathfrak{a}_{X}$ can be lifted as biholomorphisms $\tilde{\gamma}_{i}$ of $X_{\delta}^{2}$. This already implies that the composition

$$
X_{\delta}^{2} \rightarrow X_{\delta}^{1} \rightarrow X_{\delta}
$$

is a regular covering. Moreover, we can realize the monodromies $\rho_{2}(\gamma)$ for a set of elements in $\Lambda_{2}$ generating $\Gamma_{2}$ within a subgroup of automorphisms $\left\langle\hat{\gamma}_{1}, \ldots, \hat{\gamma}_{m}\right\rangle$, where $\hat{\gamma}_{j}=\exp _{1}\left(w_{j}\right)$ and $w_{j} \in \mathfrak{a}_{X_{\epsilon}^{1}}$. Since all the biholomorphisms $\tilde{\gamma}_{i}$ and $\hat{\gamma}_{j}$ are exponentials of vector fields belonging to the same Abelian algebra, they commute with each other, proving that the regular covering $X_{\delta}^{2} \rightarrow X_{\delta}$ is in fact Abelian.

If $F_{2}$ does not have nonsingular vector fields we are done. Otherwise we repeat again the above construction starting with $X_{\delta}^{2}$. Since the rank of locally free actions is bounded by the dimension $n$ of $X$, this procedure ends after a finite number of steps.

Remark 6.7. Let $\mathfrak{h}_{X}=\mathfrak{a}_{X} \oplus \mathfrak{h}_{X}^{1}$, with $\mathfrak{a}_{X}$ central, and let $X_{\epsilon}^{\prime}=F \times_{\Lambda} \mathbb{C}^{s^{\prime}}$ be as in the above proposition. Along the proof it is shown that the Lie algebra decomposition $\mathfrak{h}_{X_{\epsilon}^{\prime}}=\mathfrak{a}_{X_{\epsilon}^{\prime}} \oplus \mathfrak{h}_{X_{\epsilon}^{\prime}}^{1}$ can be taken with the property that $\mathfrak{a}_{X_{\epsilon}^{\prime}}$ is generated by the projection over $X_{\epsilon}^{\prime}$ of linear vector fields on $\mathbb{C}^{s^{\prime}}$ and the lift to $X_{\epsilon}^{\prime}$ of $\mathfrak{a}_{X}=\mathfrak{a}_{X_{\epsilon}}$ is included in $\mathfrak{a}_{X_{\epsilon}^{\prime}}$.

We are now ready to establish our refinement of Calabi's Theorem 0.2, which was originally stated by Calabi in [8] for solvable Galois group $\Gamma$. It was proved by Bogomolov in [6] for complex projective manifolds $X$ with Abelian $\Gamma$ and extended by 
Beauville [2] who showed that for a suitable finite $\Gamma$ the factor $F$ is a simply-connected manifold with special holonomy.

Corollary 6.8. Let $X$ be a compact Kähler manifold with $c_{1}(X)=0$. Then $X$ admits a finite, Abelian étale covering $X^{\prime}=F \times T$ with Galois group $\Gamma$, which is the product of a complex torus $T$ of dimension $b_{1}\left(X^{\prime}\right) / 2$ and a compact Kähler manifold $F$ with $c_{1}(F)=0$ and $b_{1}(F)=0$.

Proof. If a compact Kähler manifold has a trivial first Chern class $c_{1}(X)=0$ then the natural coupling between $\mathfrak{h}_{X}$ and $H^{0}\left(M, \Omega_{X}^{1}\right)$ is nondegenerate (cf. [26]). This implies that $\operatorname{Aut}_{\mathbb{C}}^{0}(X)$ is a complex torus, and in particular $\mathfrak{h}_{X}=\mathfrak{a}_{X}$. Therefore, in that case there is a unique locally free $\mathbb{C}^{s}$-action $\varpi$ of maximal rank $s=\operatorname{dim} \mathfrak{a}_{X}=b_{1}(X) / 2$.

The nondegeneracy of the above coupling also implies that the foliation $\mathcal{F}$ determined by the action $\varpi$ does not have basic 1 -forms. Hence $(X, \varpi)$ does not have nontrivial $b$ deformations and the approximation theorem says in that case that $X$ is already a suspension $F \times{ }_{\Lambda} \mathbb{C}^{s}$. Now the statement can be proved as the above theorem by just remarking that, at each stage, the fiber manifold $F$ also has $c_{1}(F)=0$ (cf. 2.9) and therefore there is no need to consider tangential deformations.

\section{Structure of Kähler manifolds with nonvanishing vector fields}

With the exception of Proposition 7.1, we assume from now on that the compact complex manifold $X$ endowed with a locally free $\mathbb{C}^{s}$-action is of Kähler type. As we pointed out, examples of such manifolds are (i) complex tori, (ii) flat principal torus bundles over a Kähler manifold and (iii) suspensions over a torus $T=\mathbb{C}^{s} / \Lambda$ with fiber a Kähler manifold $F$ and monodromy $\rho: \Lambda \rightarrow \operatorname{Aut}_{\mathbb{C}}^{0}(F)$.

A natural question is whether that list of examples covers all possible Kähler manifolds $X$ with locally free Abelian actions. The answer is positive, up to a finite covering, if the action has codimension one, i.e. if $s=n-1$ (as proved by F. Bosio in [7]), if $c_{1}(M)=0$ (Corollary 6.8) or if the manifold $X$ is projective (as proved in Section 3).

In this section we show that, for a general Kähler manifold, the answer is also positive but up to a finite covering and up to a (small) tangential deformation of the manifold. The first two propositions are precise statements of this fact. We also show (Examples 7.6 and 7.7) that tangential deformations cannot be avoided.

Afterwards, combining tangential deformations with deformations of representations, we are able to give a general structure theorem for Kähler manifolds with locally free Abelian actions (Theorem 7.11). From it we deduce that such a manifold $X$ has a finite covering which is a (not necessarily small) deformation of a product $F \times T$. Also as a corollary of that construction, we prove that the manifolds $X_{r}$ in the versal family $X_{R}$ of tangential deformations of $X$ are Kähler manifolds for each $r \in R$.

Assume that the Abelian group defining the action on $X$ is a complex torus. It was proved by Fujiki [16] and Lieberman [28] that, under this hypothesis, the manifold $X$ is a Seifert fibration with the torus as the typical fiber. The following statement is a slight improvement of that result. 
Proposition 7.1. Let $X$ be a compact manifold in the class $\mathcal{C}$ endowed with a locally free $\mathbb{C}^{s}$-action. Assume that all the orbits of the action are closed. Then $X$ is the quotient by an Abelian finite group of a flat torus bundle $X^{\prime}$,

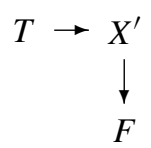

where $T$ is a complex torus of dimension $s$ and $F$ is a compact manifold in the class $\mathcal{C}$. Furthermore, there is an arbitrarily small b-deformation $X_{\epsilon}$ of $X$ such that $X_{\epsilon}$ is a finite quotient of the product manifold $F \times T$.

Proof. By Theorem 6.3, there is a small $b$-deformation $X_{\epsilon}$ of $X$ that is the suspension $F \times{ }_{\Lambda} \mathbb{C}^{s}$ over a torus $T=\mathbb{C}^{s} / \Lambda$ defined by a representation $\rho: \Lambda=\pi_{1}(T) \rightarrow \operatorname{Aut}_{\mathbb{C}}(F)$. The orbits of the action on $X_{\epsilon}$ are still closed and all orbits are finite coverings of $T$. In particular all the elements of $H=\rho(\Lambda)$ are of finite order and this implies that the group $H$ itself is finite (cf. [21]). Hence, the $\mathbb{C}^{s}$-action on $X_{\epsilon}$ defines a Seifert fibration over the complex orbifold $\hat{F}=F / H$. Notice that the natural projection $F \rightarrow \hat{F}$ is a ramified covering.

Set $T_{0}=\mathbb{C}^{s} / \Lambda_{0}$, where $\Lambda_{0}=\operatorname{ker} \rho$. The pull-back of the fibration $X_{\epsilon} \rightarrow T$ by the covering map $T_{0} \rightarrow T$ has total space an Abelian finite covering $X_{\epsilon}^{\prime}$ of $X_{\epsilon}$ naturally identified to the product $F \times T_{0}$. Indeed, since $H=\Lambda / \Lambda_{0}$ we have $X_{\epsilon}=F \times_{H} T_{0}$.

Finally, notice that there is an Abelian finite covering $X^{\prime}$ of $X$ such that $X_{\epsilon}^{\prime}$ is a $b$ deformation of $X^{\prime}$ (cf. Proposition 5.13). Then the covering space $X^{\prime}$ has the required properties.

Remark 7.2. It follows from the above proof that, under the hypothesis of the theorem, $X$ is a Seifert fibration over a good orbifold, i.e. an orbifold which is the quotient of a manifold by a finite group.

Combining previous results we can state

Proposition 7.3. Let $X$ be a compact Kähler manifold endowed with a locally free $\mathbb{C}^{s}$ action, where $s=\operatorname{dim}_{\mathbb{C}} \mathfrak{h}_{X} / \mathfrak{h}_{X}^{1}$. There is a finite Abelian covering $X_{\epsilon}^{\prime}$ of a small $b$ deformation $X_{\epsilon}$ of $X$, a torus $T$ of dimension $s^{\prime} \geq s$ and a compact Kähler manifold $F$ without nonvanishing vector fields such that

(i) if $\mathfrak{h}_{X}^{1}=0$ then $X_{\epsilon}^{\prime}=F \times T$,

(ii) if $\mathfrak{h}_{X}^{1} \neq 0$ then $X_{\epsilon}^{\prime}$ is a suspension over $T$ with fiber $F$, monodromy in $\operatorname{Aut}_{\mathbb{C}}^{0}(F)$, and $\mathfrak{h}_{F}=\mathfrak{h}_{F}^{1} \neq 0$; in particular $F$ and $X$ are uniruled manifolds.

In both cases $\operatorname{kod}(F)=\operatorname{kod}(X)$.

Proof. Recall that $\mathfrak{h}_{X}^{1}=0$ implies that $\operatorname{Aut}_{\mathbb{C}}^{0}(X)$ is a torus and in that case the orbits of the action are closed. Hence case (i) follows from Propositions 6.5 and 7.1.

Without loss of generality we can assume that the subalgebra $\mathfrak{a}$ has been chosen in the center of $\mathfrak{h}_{X}$. Then the space of vector fields with zeros is the same for every tangential 
deformation of the manifold (cf. Proposition 5.9). Therefore, we can apply again Proposition 6.5 to case (ii), and we have $\mathfrak{h}_{X_{\epsilon}}^{1} \neq 0$ and also $\mathfrak{h}_{F}^{1} \neq 0$, as vector fields with zeros are always tangent to fibers in the case of suspensions. In particular the manifolds $X$ and $F$ are both uniruled by Theorem 1.8 .

Finally, the last statement follows directly from Proposition 5.16, Corollary 2.9 and the fact that taking an (unramified) finite covering does not change the Kodaira dimension.

Remark 7.4. If $\mathfrak{h}_{X}^{1} \neq 0$ then $X$ is a uniruled manifold and therefore $\operatorname{kod}(X)=-\infty$. So there are the following possibilities according to the Kodaira dimension of $X$ :

(a) $\operatorname{kod}(X) \geq 0$. Then we are in case (i) in the above proposition and the manifold $F$ fulfills the stronger condition $\mathfrak{h}_{F}=0$, because $\operatorname{kod}(F) \geq 0$.

(b) $\operatorname{kod}(X)=-\infty$. Since the $\mathbb{C}^{s}$-action can have closed orbits both possibilities, (i) and (ii) in the above proposition, can occur.

We are now ready to complete the proof of Theorem 0.3 :

Proof of Theorem 0.3. The algebraic case follows from Theorem 3.2. The general statement follows directly from Proposition 7.3, Remark 7.4 and from the observation that, when $\operatorname{kod}(X) \geq 0$, the connected component of the identity $\operatorname{Aut}_{\mathbb{C}}^{0}(F)$ of the group of automorphisms of the fiber manifold $F$ reduces to the identity.

We give here two examples showing that, in order to obtain a complete classification up to finite coverings of Kähler manifolds with nonsingular vector fields, one cannot avoid the use of tangential deformations. More precisely, we exhibit examples of Kähler manifolds $X$ endowed with a locally free $\mathbb{C}$-action with the property that neither $X$ nor any finite covering $X^{\prime}$ of $X$ are suspensions or torus bundles. We will use the following characterization of manifolds that are suspensions over a complex torus in terms of their Albanese torus.

Proposition 7.5. Let $X$ be a compact Kähler manifold with $s=\operatorname{dim}_{\mathbb{C}} T_{X}=\operatorname{dim}_{\mathbb{C}} \mathfrak{h}_{X} / \mathfrak{h}_{X}^{1}$ $>0$. The manifold $X$ is a suspension over an s-dimensional torus $\mathbb{T}^{s}$ with a connected fiber if and only if the Albanese torus $\mathrm{Alb}(X)$ splits, up to isogeny, as a product $T_{X} \times T^{\prime}$.

Proof. Assume that $p: X \rightarrow \mathbb{T}^{s}$ is a suspension. The universal property of $\operatorname{Alb}(X)$ gives a commutative diagram

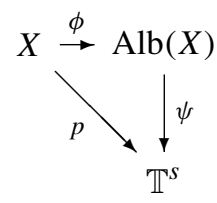

Then the restriction of $\psi$ to $T_{X}$ is a surjective group homomorphism with finite kernel and $\operatorname{Alb}(X)$ is isogenous to $T_{X} \times \operatorname{ker} \psi$.

Conversely, if $\mathrm{Alb}(X)$ is isogenous to $T_{X} \times T^{\prime}$, there is a surjective group homomorphism $\psi: \operatorname{Alb}(X) \rightarrow T_{X}$. The composition $\psi \circ \phi: X \rightarrow T_{X}$ is an immersion when restricted to the orbits of the $\mathbb{C}^{s}$-action, hence it defines $X$ as a suspension over $T_{X}$. Finally, by replacing $T_{X}$ by a finite covering of it, we can assume that the projection $\psi \circ \phi$ has connected fibers (cf. Remark 6.2). 
Example 7.6 (Examples that are not suspensions). Let $C_{g}$ be a compact Riemann surface of genus $g>1$, and $\Pi$ its $g \times 2 g$ period matrix for fixed bases of $H_{1}\left(C_{g}, \mathbb{Z}\right)$ and $H^{0}\left(C_{g}, \Omega_{C_{g}}^{1}\right)$. If $\Lambda=\Pi \cdot \mathbb{Z}^{2 g}$ is the lattice of $\mathbb{C}^{g}$ generated by the columns of the matrix $\Pi$ then $\operatorname{Alb}\left(C_{g}\right)=\mathbb{C}^{g} / \Lambda$. Let $E=\mathbb{C} /(\mathbb{Z} \oplus \mathbb{Z} \tau)$ be a fixed elliptic curve. The complex tori $T$ that are extensions of the type

$$
0 \rightarrow E \rightarrow T \rightarrow \operatorname{Alb}\left(C_{g}\right) \rightarrow 0
$$

are parametrized by the $(g+1) \times(2 g+2)$ complex matrices of the form

$$
\left(\begin{array}{ccccc}
1 & \tau & e_{1} & \ldots & e_{2 g} \\
0 & 0 & & & \\
\vdots & \vdots & & \Pi & \\
0 & 0 & & &
\end{array}\right) .
$$

Denote by $T_{e}$ the extension defined by the fixed period matrices $(1 \tau)$ and $\Pi$, and a given vector $e=\left(e_{1}, \ldots, e_{2 g}\right) \in \mathbb{C}^{2 g}$. Then $T_{e}$ is the Albanese torus of an elliptic surface $S_{e}$, which is the analytically locally trivial fibration<smiles>C=CC(C)C</smiles>

having as monodromy automorphisms the translations by $e_{1}, \ldots, e_{2 g} \in E=\mathbb{C} /(\mathbb{Z} \oplus \mathbb{Z} \tau)$ along the selected basis of $H_{1}\left(C_{g}, \mathbb{Z}\right)$. That is, $S_{e}$ is the suspension over $C_{g}$ associated to the representation $p_{e}: \pi_{1}\left(C_{g}\right) \rightarrow E$ induced by the above translations. As the monodromy is isotopic to the identity, $S_{e}$ has even first Betti number and therefore it is a Kähler surface. Notice also that the Albanese morphism $\phi_{S_{e}}: S_{e} \rightarrow T_{e}=\operatorname{Alb}\left(S_{e}\right)$ maps $S_{e}$ isomorphically onto its image $\phi_{S_{e}}\left(S_{e}\right) \subset T_{e}$, and $\phi_{S_{e}}$ identifies $E$ with the subtorus $T_{S_{e}}=\Phi\left(\operatorname{Aut}_{\mathbb{C}}^{0}\left(S_{e}\right)\right)$ of $\operatorname{Alb}\left(S_{e}\right)$ canonically associated to $S_{e}$.

The elliptic surfaces $S_{e}$ are endowed with a locally free holomorphic $\mathbb{C}$-action given by the constant tangent vector fields of the fiber $E$, as they are preserved by the monodromy automorphisms of the suspension $S_{e} \rightarrow C_{g}$. By Proposition 7.5, a necessary condition for $S_{e}$ to be a suspension over a (one-dimensional) torus is that its Albanese torus $T_{e}$ splits, up to isogeny, as a product $E \times \operatorname{Alb}\left(C_{g}\right)$. Yet it is known that the exact sequence (18) splits up to isogeny for only countably many choices modulo isomorphism equivalence of extension data, while the set of all extensions modulo isomorphism equivalence is a $g$-dimensional complex analytic variety (cf. [3]).

Therefore, a generic choice of $e \in \mathbb{C}^{2 g}$ yields a Kähler elliptic surface $S_{e}$, which has a locally free $\mathbb{C}$-action along its fibers, but is not a suspension over a torus (although it is a suspension over $C_{g}$ by construction). Suspensions over tori that are projective manifolds can be characterized in terms of their Albanese torus as stated in Proposition 8.6. In particular, by Poincaré's reducibility theorem, the manifolds $S_{e}$ so constructed are not projective and their Albanese tori $\operatorname{Alb}\left(S_{e}\right)$ are not Abelian varieties.

Finally, consider a finite covering $\tilde{S}_{e}$ of $S_{e}$. Necessarily, $\tilde{S}_{e}$ is also an $\tilde{E}$-principal fibration over a Riemann surface $\tilde{C}_{g}$, where $\tilde{E}$ and $\tilde{C}_{g}$ are finite coverings of $E$ and $C_{g}$ 
respectively. If $\tilde{S}_{e}$ were a suspension over a torus then it would be a finite quotient of a product of two Riemann surfaces and hence a projective manifold. In that case $\operatorname{Alb}\left(\tilde{S}_{e}\right)$ would be an Abelian variety and, as the natural map $\operatorname{Alb}\left(\tilde{S}_{e}\right) \rightarrow \operatorname{Alb}\left(S_{e}\right)$ is surjective, $\operatorname{Alb}\left(S_{e}\right)$ would also be an Abelian variety, leading to a contradiction.

Example 7.7 (Examples that are neither suspensions nor torus bundles). Using the above example we now construct a compact Kähler 3-fold $X$ endowed with a locally free $\mathbb{C}$-action in such a way that neither $X$ nor any of its finite coverings are a suspension over a torus or a torus bundle.

Choose a closed Riemann surface $C_{g}$, an elliptic curve $E$ and a Kähler elliptic surface $S_{e}$ which is not a suspension over a torus, as in Example 7.6. Let $L \in \operatorname{Pic}^{0}(E)$ be a flat line bundle over $E$ which is nontorsion. Sum $L$ with the trivial local system to get a rank 2 one, $V=\mathbb{C} \oplus L$, and let $Y=\mathbb{P}_{E}(V)$ be the ruled surface over $E$ obtained by projectivizing the holomorphic rank 2 vector bundle defined by $V \otimes \mathcal{O}_{E}$.

The ruled surface $Y$ admits a locally free holomorphic $\mathbb{C}$-action, given by parallel transport along the flat connection on $V$, with the property that the projection $Y \rightarrow E$ is equivariant when we consider on $E$ the natural $\mathbb{C}$-action. It induces a group morphism $v: \mathbb{C} \rightarrow \operatorname{Aut}_{\mathbb{C}}^{0}(Y)$.

The monodromy morphism $p_{e}: \pi_{1}\left(C_{g}\right) \rightarrow E$ defining the elliptic surface $S_{e}$ of Example 7.6 can be lifted to a morphism $\tilde{p}_{e}: \pi_{1}\left(C_{g}\right) \rightarrow \mathbb{C}$ from $\pi_{1}\left(C_{g}\right)$ to the universal covering $\mathbb{C}$ of $E$. Then the representation

$$
\pi_{1}\left(C_{g}\right) \stackrel{\tilde{p}_{e}}{\rightarrow} \mathbb{C} \stackrel{\nu}{\rightarrow} \operatorname{Aut}_{\mathbb{C}}^{0}(Y)
$$

defines an analytically locally trivial fibration $X$ which is a suspension over $C_{g}$ with fiber $Y$

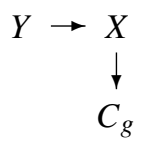

and it follows from Blanchard's criterion [5, Théorème principal II] that $X$ is a Kähler manifold. Notice that the 3-fold $X$ is naturally endowed with a locally free holomorphic $\mathbb{C}$-action, coming from the flat parallel transport of $Y$ over $E$, which is invariant under translations in $E$ and may therefore be glued under the monodromy $v \circ \tilde{p}_{e}$.

Each holomorphic vector field on $Y$ projects over $E$, hence there is a natural surjective morphism $\eta: \operatorname{Aut}_{\mathbb{C}}^{0}(Y) \rightarrow \operatorname{Aut}_{\mathbb{C}}^{0}(E)=E$ satisfying

$$
p_{e}=\eta \circ v \circ \tilde{p}_{e}
$$

so we may glue the projections $Y \rightarrow E$ to get a commutative diagram of analytically locally trivial fibrations

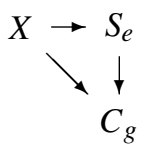

The fibration $X \rightarrow S_{e}$ has fiber $\mathbb{C} P^{1}$, so it induces an isomorphism $\operatorname{Alb}(X) \cong \operatorname{Alb}\left(S_{e}\right)$ and an identification of the Albanese images $\phi_{X}(X)=\phi\left(S_{e}\right)$. Therefore the subtorus 
$E \subset \operatorname{Alb}(X)$ does not have a complementary subtorus, even up to isogeny, and $X$ is not a suspension. In particular the manifolds $X$ and $\operatorname{Alb}(X)$ are not algebraic.

Moreover, two choices of local systems $V=\mathbb{C} \oplus L, V^{\prime}=\mathbb{C} \oplus L^{\prime}$ yield isomorphic ruled surfaces $Y$ and $Y^{\prime}=\mathbb{P}_{E}\left(V^{\prime}\right)$ if and only if $L^{\prime} \cong L^{\otimes \pm 1}$ (cf. [1, III.7]). Therefore, the choice of a nontorsion line bundle $L$ ensures that the orbits of the $\mathbb{C}$-action on $Y$ do not cover $E$ with finite degree, and this implies that neither $Y$ nor $X$ can have the structure of a torus bundle.

We remark finally that each unramified finite covering of $X$ has the same properties. Indeed, a finite covering $\tilde{X}$ of $X$ is necessarily an analytically locally trivial fibration over a Riemann surface $\tilde{C}_{g}$ with fiber a ruled surface $\tilde{Y}$ such that $\tilde{C}_{g}$ and $\tilde{Y}$ are finite coverings of $C_{g}$ and $Y$ respectively. Moreover, there is a finite covering $q: \tilde{E} \rightarrow E$ such that $\tilde{Y}$ is the projectivization $\mathbb{P}_{\tilde{E}}\left(V^{\prime}\right)$ of the rank 2 bundle $V^{\prime}=q^{*} V=\mathbb{C} \oplus q^{*} L$. Since the line bundle $q^{*} L$ over $\tilde{E}$ is also nontorsion, the manifolds $\tilde{Y}, \tilde{X}$ cannot have the structure of a torus bundle. The fact that $X^{\prime}$ cannot be a suspension over a torus follows from the same argument used to discuss the previous example.

In order to describe more accurately the structure of Kähler manifolds $X$ having nonsingular vector fields, in particular those satisfying $\mathfrak{h}_{X}^{1}>0$, we introduce here a more general class of deformations that combines the notion of tangential deformation introduced above with that of deformation of representations.

In what follows we assume that a lattice $\Lambda$ of $\mathbb{C}^{s}$, as well as a set of generators of it, $\gamma_{1}, \ldots, \gamma_{2 s}$, have been fixed. We write $\gamma_{j}=\exp _{1}\left(\tilde{v}_{j}\right)$ with $\tilde{v}_{j}$ a linear combination

$$
\tilde{v}_{j}=\sum_{i=1}^{s} B_{j}^{i} v_{i}
$$

of the coordinate vector fields $v_{1}, \ldots, v_{s}$ of $\mathbb{C}^{s}$ and where $\exp _{t}(w)$ stands for the exponential of a vector field $w$ at time $t$.

Let us consider the product $\tilde{Z}=F \times \mathbb{C}^{s}$ where $F$ is a fixed compact Kähler manifold. We assume that $F$ has no vector fields without zeros and we fix an Abelian subalgebra $\mathfrak{b}$ of $\mathfrak{h}_{F}^{1}=\mathfrak{h}_{F}$ (we do not exclude the case $\mathfrak{b}=0$ ). We think of the coordinate vector fields $v_{1}, \ldots, v_{s}$ of $\mathbb{C}^{s}$ as defined on the product $\tilde{Z}=F \times \mathbb{C}^{s}$ and, in the same way, we identify each $w \in \mathfrak{b}$ with the vector field $(w, 0)$ on $\tilde{Z}$. Then $\mathfrak{b}$ and the vector fields $v_{i}$ generate an

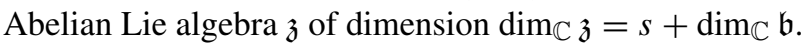

We fix vector fields $w_{1}, \ldots, w_{2 s}$ in $\mathfrak{b}$ and define $\varphi^{j}=\exp _{1}\left(\tilde{v}_{j}+w_{j}\right)$ for $j=$ $1, \ldots, 2 s$. Then $\varphi^{1}, \ldots, \varphi^{2 s}$ are commuting biholomorphisms of $\tilde{Z}$ defining a free $\mathbb{Z}^{2 s}$ action on $\tilde{Z}$. The quotient manifold $Z$ is naturally endowed with the locally free $\mathbb{C}^{s}$-action induced by the projection of the vector fields $v_{i}$. Notice that $Z$ is a suspension over the torus $\mathbb{C}^{S} / \Lambda$ with fiber $F$. If the vector fields $w_{j}$ are all zero, then the manifold $Z$ is just the product $F \times \mathbb{C}^{s} / \Lambda$.

We now generalize the above construction of the manifold $Z$ in the following way. We denote by $\alpha^{1}, \ldots, \alpha^{s}$ the dual basis of $v_{1}, \ldots, v_{s}$, also thought of as holomorphic 1 -forms on $\tilde{Z}$. We consider the tangential deformations of the $\mathbb{C}^{s}$-action on $\tilde{Z}$ as defined in Section 5. That is, for a given $r=(C, \theta) \in \Xi=$ End $\mathbb{C}^{s} \oplus\left\langle\alpha^{1}, \ldots, \alpha^{s}\right\rangle$, we define $\alpha_{r}^{i}$ 
as in (10), we denote by $R$ the subset of $\Xi$ satisfying the conditions stated in Definition 5.1 and we define new vector fields $v_{i}^{r}$ by the condition $v_{i}^{r}=C v_{i}^{\prime}$, where $v_{i}^{\prime}$ are the vector fields on $\tilde{Z}$ that are tangent to the $\mathbb{C}^{s}$-action and are determined by the conditions $\alpha_{r}^{i}\left(v_{j}^{\prime}\right)=\delta_{j}^{i}$ and $\alpha_{r}^{i}\left(\overline{v_{j}^{\prime}}\right)=0$.

We denote by $\tilde{Z}_{r}$ the product $F \times \mathbb{C}^{s}$ endowed with this new complex structure and with the holomorphic $\mathbb{C}^{s}$-action determined by the vector fieds $v_{i}^{r}$. Notice that $\mathfrak{b}$ and $v_{1}^{r}, \ldots, v_{s}^{r}$ generate an Abelian Lie algebra $\mathfrak{z} r$ of holomorphic vector fields on $\tilde{Z}_{r}$. Finally, we consider the vector fields $\tilde{v}_{1}^{r}, \ldots, \tilde{v}_{2 s}^{r}$ on $\tilde{Z}_{r}$ defined as

$$
\tilde{v}_{j}^{r}=\sum_{i=1}^{s} B_{j}^{i} v_{i}^{r}
$$

Definition 7.8. Let $\Lambda$ be a fixed lattice of $\mathbb{C}^{s}$. Let $F$ be a compact Kähler manifold without nonsingular vector fields and let $\mathfrak{b}$ be an Abelian subalgebra of $\mathfrak{h}_{F}^{1}=\mathfrak{h}_{F}$. Fix vector fields $w_{1}, \ldots, w_{2 s}$ in $\mathfrak{b}$, and for a given $u=\left(a_{1}, \ldots, a_{2 s}\right)$ in $\mathbb{C}^{2 s}$ and $r \in R$, define the commuting automorphisms $\varphi_{u, r}^{j}$ of $\tilde{Z}_{r}$ by

$$
\varphi_{u, r}^{j}=\exp _{1}\left(\tilde{v}_{j}^{r}+a_{j} w_{j}\right)
$$

where $\tilde{v}_{j}^{r}$ are the vector fields defined in (20). Let $S$ be the subset of $\mathbb{C}^{2 s} \times R$ of those pairs $(u, r)$ such that the $\mathbb{Z}^{2 s}$-action on $\tilde{Z}_{r}$ defined by the automorphisms $\varphi_{u, r}^{j}$ is free. In that case we denote by $Z_{u, r}$ the quotient manifold.

The family $Z_{S}=\left\{Z_{u, r}\right\}$ is a holomorphic family of deformations of compact complex manifolds endowed with locally free $\mathbb{C}^{S}$-actions parametrized by $S$.

Remarks 7.9. (a) If $(u, r)$ is an element of $S$ then $(t \cdot u, r)$ also belongs to $S$ for each $t \in[0,1]$, hence $S$ is a connected set containing a neighborhood of $R \cong\{0\} \times R$. The restriction of the family $Z_{S}$ to $\{0\} \times R$ is just the product of $F$ with the family of complex tori.

(b) The manifolds $Z_{u, r}$ are not necessarily suspensions over a torus. In fact each (small) tangential deformation of $Z_{u, r}$ is an element of $Z_{S}$.

Proposition 7.10. The complex manifolds $Z_{u, r}$ of the family $Z_{S}$ defined above are Kähler manifolds for each $(u, r) \in S$.

Proof. The manifolds $Z_{0, r}$, being products of $F$ with complex tori, are all Kähler manifolds. The stability theorem of Kodaira implies that $Z_{u, r}$ are also Kähler manifolds for $u$ small enough. Now let an element $Z_{u, r}$ of the family $Z_{S}$ be given. We notice that $Z_{u, r}$ is a finite covering of the complex manifold $Y_{m}$ defined as the quotient of $\tilde{Z}_{r}$ by the automorphisms

$$
\psi^{j}=\exp _{1 / m}\left(\tilde{v}_{j}^{r}+a_{j} w_{j}\right)=\exp _{1}\left(\frac{1}{m} \tilde{v}_{j}^{r}+\frac{a_{j}}{m} w_{j}\right)
$$

where $m$ is a positive integer. Hence it suffices to prove that $Y_{m}$ is a Kähler manifold. But the map $h: F \times \mathbb{C}^{s} \rightarrow F \times \mathbb{C}^{s}$ defined by $h(z, t)=(z, m t)$ is a holomorphic 
automorphism of $\tilde{Z}_{r}$ that induces a biholomorphism from $Y_{m}$ onto $Z_{u^{\prime}, r}$, where $u^{\prime}=u / m$. Since $m$ can be taken arbitrarily large, $Z_{u^{\prime}, r}$ is arbitrarily close to $Z_{0, r}$, and this ends the proof.

Now, we are able to prove the main result of this section:

Theorem 7.11. Let $X$ be a compact Kähler manifold with nonsingular vector fields. There is a finite Abelian covering $X^{\prime}$ of $X$, a lattice $\Lambda$ of $\mathbb{C}^{s}$, a compact Kähler manifold $F$ with $\mathfrak{h}_{F}^{1}=\mathfrak{h}_{F}$ and vector fields $w_{1}, \ldots, w_{2 s}$ in an Abelian subalgebra $\mathfrak{b}$ of $\mathfrak{h}_{F}$ such that $X^{\prime}$ is biholomorphic to a manifold $Z_{u, r}$ in the family $Z_{S}$ constructed above for a suitable pair $(u, r) \in S$.

Proof. We know the existence of the covering $X^{\prime}$ of $X$, a small tangential deformation $X_{\epsilon}^{\prime}$ of $X^{\prime}$ and a compact Kähler manifold $F$ without nonvanishing vector fields such that $X_{\epsilon}^{\prime}$ is the suspension over a torus $T=\mathbb{C}^{s} / \Lambda$ associated to a representation $\rho: \Lambda \rightarrow \operatorname{Aut}_{\mathbb{C}}^{0}(F)$ (cf. Proposition 6.5). Remark that it is sufficient to prove that $X_{\epsilon}^{\prime}$ belongs to the family $Z_{S}$.

If $\rho$ reduces to the identity then we are in case (i) of Proposition 7.3 and the assertion is clear as $X_{\epsilon}^{\prime}=F \times T$. So, assume that $\rho$ is not constant and let $\gamma_{1}, \ldots, \gamma_{2 s}$ be a set of generators of $\Lambda$. Notice that $\operatorname{Aut}_{\mathbb{C}}^{0}(F)$ is an algebraic group. This is a consequence of the Fujiki-Lieberman theorem because $\mathfrak{h}_{F}=\mathfrak{h}_{F}^{1}$. Therefore the Zariski closure $B$ of $\rho(\Lambda)$ in $\operatorname{Aut}_{\mathbb{C}}^{0}(F)$ is an Abelian algebraic group and in particular it has a finite number of connected components. So, replacing $X^{\prime}$ by an Abelian finite covering if necessary, we can assume that the group $B$ is connected. Let $\mathfrak{b}$ be the Lie algebra of $B$. As connected Abelian Lie groups are exponential, we can write $\rho\left(\gamma_{j}\right)=\exp _{1}\left(w_{j}\right)$ for $j=1, \ldots, 2 s$ and suitable vector fields $w_{j} \in \mathfrak{b}$. Then the lattice $\Lambda$, the Kähler manifold $F$, the Abelian Lie algebra $\mathfrak{b}$ and the vector fields $w_{1}, \ldots, w_{2 s}$ fulfill the required conditions.

As a corollary we obtain

Corollary 7.12. Let $X$ be a compact Kähler manifold endowed with a locally free $\mathbb{C}^{s}$ action. Then there is a finite Abelian covering $X^{\prime}$ of $X$, which is a deformation of the product $F \times T$ of a Kähler manifold $F$ without nonvanishing vector fields and a complex torus $T$.

Remark 7.13. Notice that $s=\operatorname{dim}_{\mathbb{C}} T_{X}=\operatorname{dim}_{\mathbb{C}} \mathfrak{h}_{X} / \mathfrak{h}_{X}^{1}$ can be smaller than the dimension of $T$.

We can now prove Theorem 5.17, i.e. if $X$ is a Kähler manifold then each $X_{r}$ in the versal family $X_{R}$ of tangential deformations of $(X, \varpi)$ is also a Kähler manifold.

Proof of Theorem 5.17. Let $r \in R$ be given. An easy computation shows that if $r^{\prime}$ is close enough to (id, 0 ) then $X_{r}$ can be identified to an element of the versal family $\left(X_{r^{\prime}}\right)_{R}$ of $X_{r^{\prime}}$. Hence we can assume without loss of generality that the Kähler manifold $X$ is already a suspension over a torus $T=\mathbb{C}^{S} / \Lambda$. Since finite coverings and finite quotients of Kähler manifolds are Kähler manifolds too, we can also assume that $X$ is the suspension over $T$ of a representation $\rho: \Lambda \rightarrow \operatorname{Aut}_{\mathbb{C}}^{0}(F)$, where $F$ is a Kähler manifold without nonsingular vector fields. 
As in the proof of Theorem 7.11, we can write $\rho\left(\gamma_{j}\right)=\exp _{1}\left(w_{j}\right)$, where $\gamma_{1}, \ldots, \gamma_{2} s$ are a set of generators of $\Gamma$ and $w_{j}$ are suitable vector fields in an Abelian subalgebra $\mathfrak{b}$ of $\mathfrak{h}_{F}^{1}$. Then, if we set $\gamma_{j}=\exp _{1}\left(\tilde{v}_{j}\right)=\exp _{1}\left(\sum B_{j}^{i} v_{i}\right)$ as in (19), the manifold $X$ is the quotient of $\tilde{Z}=F \times \mathbb{C}^{s}$ by the Abelian group generated by the automorphisms $\varphi^{j}=\exp _{1}\left(\tilde{v}_{j}+w_{j}\right)$. Hence $X$ is an element of the family $Z_{S}$ associated to $\Lambda, F$ and the commuting vector fields $w_{j}$ on $F$. We claim that $X_{r}$ also belongs to the family $Z_{S}$. Indeed, $X_{r}$ is the quotient of $\tilde{Z}$ by the group generated by the automorphisms

$$
\exp _{1}\left(\sum B_{j}^{i} v_{j}^{r}+w_{j}\right)
$$

that is, $X_{r}$ coincides with the manifold $Z_{u_{1}, r}$ where $u_{1}=(1, \ldots, 1)$. Now the statement is a consequence of Proposition 7.10.

\section{Locally free $\mathbb{C}^{S}$-actions on Kähler manifolds with small codimension}

In this section we classify compact Kähler manifolds $X$ endowed with a locally free holomorphic $\mathbb{C}^{s}$-action when the codimension of the action is $n-s \leq 2$. Recall that, by inequality (3), we have $\operatorname{kod}(X) \leq n-s$.

If $s=n$ the manifold $X$ is a quotient of $\mathbb{C}^{s}$, hence we have (notice that the hypothesis of being Kähler is not needed here)

Proposition 8.1. Assume that $s=n=\operatorname{dim}_{\mathbb{C}} X$. Then $X$ is a complex torus.

The case $s=n-1$ was first discussed by Bosio (cf. [7]). The following statement is a slight improvement of Bosio's result. We give here an alternative and simpler proof.

Proposition 8.2 (Bosio). Assume that $s \geq n-1$.

(i) If $\operatorname{kod}(X)=1$ then $X$ has a finite Abelian covering $X^{\prime}$ which is an $(n-1)$-torus bundle over a Riemann surface $C_{g}$ of genus $g \geq 2$. Moreover, there is a torus $T$ and a small $b$-deformation $X_{\epsilon}^{\prime}$ of $X^{\prime}$ such that $X_{\epsilon}^{\prime}=C_{g} \times T$.

(ii) If $\operatorname{kod}(X)=0$ then $X$ has a finite Abelian covering $X^{\prime}$ which is a torus.

(iii) If $\operatorname{kod}(X)=-\infty$ then $X$ is a suspension over a torus $T$ with fiber $\mathbb{C} P^{1}$; that is, $X$ is a flat ruled manifold over $T$. In this case $\mathfrak{h}_{X}^{1} \neq 0$.

Proof. Notice that a small $b$-deformation $X_{\epsilon}$ of $X$ is a suspension over a torus $T$ with a fiber $F$ that can be a Riemann surface of genus $g \geq 2$, an elliptic surface or $\mathbb{C} P^{1}$. As $\operatorname{kod}(F)=\operatorname{kod}\left(X_{\epsilon}\right)=\operatorname{kod}(X)$ these three cases correspond respectively to $\operatorname{kod}(X)$ equal to 1,0 or $-\infty$.

The first two statements follow directly from Proposition 7.1 (cf. Remark 7.4).

If $\operatorname{kod}(X)=-\infty$ then $F=\mathbb{C} P^{1}$. But, since in this case $b_{1}(F)=0$, the manifold $X$ has no $b$-deformations and $X=X_{\epsilon}$. Hence $X$ is a flat ruled manifold over $T$. Notice finally that, as the monodromy $\rho: \pi_{1}(T) \rightarrow \operatorname{PGL}(2, \mathbb{C})$ defining the suspension is Abelian, it fixes a vector field of $\mathbb{C} P^{1}$. This proves that $\mathfrak{h}_{X}^{1} \neq 0$. 
Remark 8.3. Let $X$ be a compact complex manifold in the class $\mathcal{C}$ endowed with a locally free $\mathbb{C}^{s}$-action. If $n-s \leq 1$ then $X$ is a Kähler manifold. Indeed, if $s=n$ Proposition 8.1 applies and $X$ is a torus. If $s=n-1$ then a small tangential deformation $X_{\epsilon}$ is a suspension over a torus $T$ with fiber a Riemann surface $C$. Since $b_{2}(C)=1$, the suspension $X_{\epsilon}$ is a Kähler manifold (cf. [29, Corollary 3.21]), and Theorem 5.17 implies that so is $X$.

Proposition 8.4. Assume that $s \geq n-2$.

(I) If $\operatorname{kod}(X) \geq 0$ then $X$ is an $(n-2)$-torus bundle over a Kähler surface $F$. Moreover, there is a torus $T$, a finite Abelian covering $X^{\prime}$ of $X$ and a small b-deformation $X_{\epsilon}^{\prime}$ of $X^{\prime}$ such that $X_{\epsilon}^{\prime}=F \times T$. If $b_{1}(F)=0$ then $X^{\prime}=F \times T$. Furthermore:

(i) If $\operatorname{kod}(X)=2$ then $F$ is a surface of general type.

(ii) If $\operatorname{kod}(X)=1$ then $F$ is an elliptic surface.

(iii) If $\operatorname{kod}(X)=0$ then the minimal model of $F$ is a $K 3$ surface or a torus. If $F$ is a torus then $X$ is a quotient of a torus.

(II) If $\operatorname{kod}(X)=-\infty$ then the manifold $X$ is uniruled and it has a finite Abelian covering $X^{\prime}$ which belongs to one of the following types:

(i) A suspension of a group representation $\rho: \pi_{1}(T) \rightarrow \operatorname{Aut}_{\mathbb{C}}^{0}(F)$ where $F$ is a rational surface.

(ii) A small b-deformation of the suspension of a group representation $\rho: \pi_{1}(T) \rightarrow$ $\operatorname{Aut}_{\mathbb{C}}^{0}(F)$ where $F$ is a ruled surface over a Riemann surface of genus $g \geq 1$. In this case the manifold $X^{\prime}$ is ruled.

Proof. Let us consider a small $b$-deformation $X_{\epsilon}$ of $X$ which is a suspension over a torus $T$ with fiber a Kähler surface $F$. Then $\operatorname{kod}(F)=\operatorname{kod}(X)$.

Assume first that $\operatorname{kod}(X) \geq 0$. In that case the statements follow from Proposition 7.1 and Remark 7.4. We just notice that if $\operatorname{kod}(X)=0$ then $F$ is a Kähler surface whose minimal model $F_{0}$ can be a $K 3$ surface, a torus, an Enriques surface or a bielliptic surface. Since Enriques surfaces and bielliptic surfaces are Abelian quotients of K3 surfaces and torus respectively, by considering an appropriate finite covering one can assume that $F_{0}$ is of one of the first two types.

Suppose now that $\operatorname{kod}(X)=-\infty$. A small $b$-deformation $X_{\epsilon}^{\prime}$ of an Abelian finite covering $X^{\prime}$ of $X$ is the suspension over a torus $T$ associated to a representation $\rho$ : $\pi_{1}(T) \rightarrow \operatorname{Aut}_{\mathbb{C}}^{0}(F)$, where $\operatorname{kod}(F)=-\infty$. Thus, $F$ is either a rational surface or a ruled surface over a Riemann surface of genus $g \geq 1$.

If $F$ is rational, $b_{1}(F)=0$ and the manifold $X_{\epsilon}$ admits no $b$-deformations, therefore $X=X_{\epsilon}$.

If $F$ is a ruled surface then there is a geometrically ruled surface $\hat{F}$ obtained by recursively blowing down rational $(-1)$-curves of $F$. The set of $(-1)$-curves is discrete, so any element of $\operatorname{Aut}_{\mathbb{C}}^{0}(F)$ fixes them. Therefore there is a well defined group morphism $\operatorname{Aut}_{\mathbb{C}}^{0}(F) \rightarrow \operatorname{Aut}_{\mathbb{C}}^{0}(\hat{F})$. The composition of the monodromy $\rho$ with that morphism defines a suspension manifold $\hat{X}_{\epsilon}^{\prime}$ with the following two properties: (i) it is geometrically ruled and (ii) it is obtained from $X_{\epsilon}^{\prime}$ by recursively contracting the families of (-1)-curves. In particular $X_{\epsilon}^{\prime}$ is bimeromorphic to $\hat{X}_{\epsilon}^{\prime}$ and ruled. Moreover the nontrivial fibers of the contraction $X_{\epsilon}^{\prime} \rightarrow \hat{X}_{\epsilon}^{\prime}$ are transverse to the action. This allows us to define a tangential 
deformation $\hat{X}^{\prime}$ of $\hat{X}_{\epsilon}^{\prime}$ so that it is dominated by the tangential deformation $X^{\prime}$ of $X_{\epsilon}^{\prime}$. By Proposition 5.14 the manifold $\hat{X}^{\prime}$ is geometrically ruled and so $X^{\prime}$ is ruled.

In the case of $\operatorname{kod}(X)=-\infty$ we derive the following consequence:

Theorem 8.5. Assume that $s \geq n-2$ and, in the case $s=n-2$, suppose also that $\operatorname{kod}(X)=-\infty$. Then there is an arbitrarily small tangential deformation $X_{\epsilon}$ of $X$ which is a projective manifold.

In order to prove the theorem we begin by characterizing when a suspension over a torus is a projective manifold. The following proposition is a direct consequence of a result by A. Blanchard [5, p. 198] since a manifold is projective if and only if a given (unramified) covering of it is projective.

Proposition 8.6. Let $F$ be a compact Kähler manifold and $T=\mathbb{C}^{s} / \Lambda$ a complex torus. Let $X$ be the suspension of a group representation $\rho: \pi_{1}(T) \rightarrow \operatorname{Aut}_{\mathbb{C}}(F)$ and assume that it is a Kähler manifold. The following conditions are equivalent:

(i) $X$ is projective.

(ii) $F$ and $\operatorname{Alb}(X)$ are projective.

In that case, the complex torus $T$ is projective.

Remark 8.7. An easy computation shows that if $\rho(\Lambda) \subset \operatorname{Aut}_{\mathbb{C}}^{0}(F)$ then $\operatorname{Alb}(X)$ is isomorphic to the suspension over the torus $T$ of the group representation $\rho^{\prime}=\Phi \circ \rho$ : $\pi_{1}(T) \rightarrow \operatorname{Alb}(F)$, where $\Phi$ is the group morphism in the exact sequence (1).

Proposition 8.8. Let $F$ be a compact projective manifold with $\mathfrak{h}_{F} / \mathfrak{h}_{F}^{1}=0$. A Kähler manifold X obtained by suspension over a torus $T$ of a group representation $\rho: \pi_{1}(T) \rightarrow$ $\operatorname{Aut}_{\mathbb{C}}(F)$ is projective if and only if $T$ is projective.

Proof. We can assume that $\rho(\Lambda) \subset \operatorname{Aut}_{\mathbb{C}}^{0}(F)$. As $F$ admits no vector fields without zeros, the map $\Phi$ is identically zero, therefore $\operatorname{Alb}(X)=\operatorname{Alb}(F) \times T$.

Proof of Theorem 8.5. By Proposition 6.5 there is a small tangential deformation $X_{\tau}^{\prime}$ of a finite covering $X^{\prime}$ of $X$ which is a suspension over a torus $T$ and has a fiber $F$ satisfying $\mathfrak{h}_{F} / \mathfrak{h}_{F}^{1}=0$. With the hypothesis made, $F$ is a projective manifold and the above Proposition applies.

A $t$-deformation $X_{\epsilon}^{\prime}$ of $X_{\tau}^{\prime}$ is still a suspension with the same fiber $F$ and over a suitable deformation $T_{\epsilon}$ of the torus $T$ (cf. Example 5.12). As Abelian varieties are dense in the space of complex tori, we can assume that $T_{\epsilon}$ is projective and in that case $X_{\epsilon}^{\prime}$ is projective too. Finally, $X_{\epsilon}^{\prime}$ is a finite covering of a tangential deformation $X_{\epsilon}$ of $X$ which is also an algebraic manifold.

In the case of projective manifolds with $\operatorname{kod}(X) \geq 0$ we can replace the use of tangential deformations by Theorem 3.2 and we obtain a more refined classification: 
Corollary 8.9. Let $X$ be a complex projective manifold of dimension $n$, with $\operatorname{kod}(X) \geq 0$, and admitting a locally free holomorphic $\mathbb{C}^{s}$-action with $s \geq n-2$. Up to a finite, étale, Abelian covering, $X$ is either of the following:

(i) $T^{n}$,

(ii) a product $C_{g} \times T^{n-1}$ with $C_{g}$ a closed Riemann surface of genus $g \geq 2$,

(iii) a product $F \times T^{n-2}$ with $F$ a complex projective surface with $\operatorname{kod}(F)=\operatorname{kod}(X)$ and no tangent vector fields,

with $T^{k}$ denoting an Abelian variety of dimension $k$.

Applying the classification of compact complex surfaces to Corollary 8.9 we derive the following

Corollary 8.10. Conjecture 0.4 is true for projective manifolds $X$ with a locally free holomorphic $\mathbb{C}^{s}$-action of rank $s \geq \operatorname{dim}_{\mathbb{C}} X-2$.

Proof. By Corollary 8.9, such an $X$ admits as a finite étale cover either an Abelian variety, or a product $F \times T^{n-2}$ with $T^{n-2}$ another Abelian variety and $F$ a surface with $\operatorname{kod}(F)=0$. By the Kodaira-Enriques classification of surfaces, $F$ can be an Abelian, hyperelliptic, K3 or Enriques surface. In the first two cases, a finite étale cover becomes an Abelian variety. In the last two cases, an étale cover of degree 1 or 2 becomes a torus times a simply connected $F^{\prime}$ with $\operatorname{kod}(F)=0$.

\section{Dynamics of holomorphic vector fields}

A tangent vector field on a manifold defines a 1-parameter flow, consisting of biholomorphisms if both are complex analytic. The flow is complete when the manifold is compact.

Consider the continuous dynamical system $(X, v)$, formed by a compact Kähler manifold $X$ and a holomorphic tangent vector field $v$ on $X$. The classification of compact Kähler manifolds with tangent vector fields provided in Theorem 0.3 and Proposition 7.3 may be applied to study the dynamics of such dynamical systems. This is a refinement of the study carried out by D. Lieberman in [27] and [28]. The conclusion is that their dynamics reduces to the case of an Abelian Lie group action on a rational variety, i.e. on a variety bimeromorphic to $\mathbb{C} P^{n}$.

The classification of these dynamical systems is based on the decomposition described in Propositions 1.4 and 1.6 of the Lie algebra $\mathfrak{h}_{X}$ of holomorphic vector fields on $X$ as a direct sum

$$
\mathfrak{h}_{X}=\mathfrak{h}_{X}^{1} \oplus \mathfrak{a}_{X}
$$

where $\mathfrak{h}_{X}^{1}$ is the subalgebra of tangent vector fields with zeros and $\mathfrak{a}_{X}$ is a maximal rank Abelian subalgebra of vector fields linearly independent at every $x \in X$, which has been chosen in the center of $\mathfrak{h}_{X}$. Recall that, by Fujiki's Theorem 1.8, if $\mathfrak{h}_{X}^{1} \neq 0$ then $X$ is uniruled.

In the case of compact Kähler manifolds $X$ with $\mathfrak{h}_{X}^{1}=0$, Theorem 4.4 in [28] establishes that the dynamical system $(X, v)$ is integrable, in the sense that $X$ admits a Seifert fibration by tori defined by the closures of the orbits of the tangent vector fields. Proposition 7.3 allows us to make the above result more precise: 
Corollary 9.1. Let $X$ be a compact Kähler manifold with $\mathfrak{h}_{X}^{1}=0$ (e.g., if $\left.\operatorname{kod}(X) \geq 0\right)$, and let $v$ be a holomorphic tangent vector field on it. There exists a finite, Abelian, unramified cover $\tilde{X} \rightarrow X$ such that the dynamical system $(\tilde{X}, \tilde{v})$, where $\tilde{v}$ is the lift of $v$ to $\tilde{X}$, is real-analytically conjugate to $\left(F \times T, v^{\prime}\right)$, where $F$ is a compact Kähler manifold, $T$ is a complex torus, and $v^{\prime}$ is a linear vector field on $T$.

If $X$ is a complex projective manifold then $(\tilde{X}, \tilde{v})$ is biholomorphic to $\left(F \times T, v^{\prime}\right)$.

Proof. Under the hypothesis $\mathfrak{h}_{X}^{1}=0$, the group $\operatorname{Aut}_{\mathbb{C}}^{0}(X)$ is a torus and the vector field $v$ is nonsingular. By Proposition 7.3, $X$ admits a small $b$-deformation $X_{\epsilon}$ and there is a finite Abelian covering $\tilde{X}_{\epsilon} \rightarrow X_{\epsilon}$ such that $\tilde{X}_{\epsilon}=F \times T$ where $T$ is a torus and $F$ does not have vector fields without zeros. Recall that $\tilde{X}_{\epsilon}$ can be seen as a $b$-deformation of a finite covering $\tilde{X}$ of $X$. Moreover $\tilde{X}_{\epsilon}$ is real-analytically isomorphic to $\tilde{X}$ (cf. 6.4(a)). Notice also that, by Proposition 5.9, the Lie algebra $\mathfrak{h}_{X_{\epsilon}}$ coincides with $\mathfrak{h}_{X}=\mathfrak{a}_{X}$. As noted in Remark 6.7, the commutative algebra $\mathfrak{a}_{X}$ of vector fields on $X_{\epsilon}$ lifts to a subalgebra of $\mathfrak{a}_{\tilde{X}_{\epsilon}}$, the maximal Abelian algebra of nonvanishing vector fields on $\tilde{X}_{\epsilon}$ formed by the vector fields that are tangent to the factor $T$. This proves the corollary. Notice that the last statement follows from Theorem 3.2.

We now consider the case $\mathfrak{h}_{X}^{1} \neq 0$.

Theorem 9.2. Let $X$ be a compact Kähler manifold with $\mathfrak{h}_{X}^{1} \neq 0$ and let $v$ be a nonvanishing holomorphic tangent vector field on it. There exists a finite, Abelian, unramified cover $\tilde{X} \rightarrow X$ such that, if $\tilde{v}$ is the lift to $\tilde{X}$ of $v$, the dynamical system $(\tilde{X}, \tilde{v})$ is realanalytically conjugate to $\left(\tilde{X}_{\epsilon}, \tilde{v}^{\prime}+\tilde{w}\right)$, where:

(i) $\tilde{X}_{\epsilon}$ is a small b-deformation of $\tilde{X}$ which is a suspension $F \times_{\Lambda} \mathbb{C}^{s}$ over a compact torus $T=\mathbb{C}^{s} / \Lambda$, with fiber an uniruled compact Kähler manifold $F$ without nonsingular vector fields.

(ii) There is a linear algebraic subgroup $G \subset \operatorname{Aut}_{\mathbb{C}}^{0}(F)$, with $G \cong \mathbb{C}^{p} \oplus\left(\mathbb{C}^{*}\right)^{q}$, such that the monodromy of the suspension $\rho: \pi_{1}(T) \cong \Lambda \rightarrow \operatorname{Aut}_{\mathbb{C}}^{0}(F)$ has values in $G$.

(iii) $\tilde{w} \in \operatorname{Lie}(G) \subset \mathfrak{h}_{F}^{1}$.

(iv) The lift of the vector field $\tilde{v}^{\prime}$ from $F \times{ }_{\Lambda} \mathbb{C}^{s}$ to its cover $F \times \mathbb{C}^{s}$ is a linear vector field in $\mathbb{C}^{S}$.

(v) $\left[\tilde{v}^{\prime}, \tilde{w}\right]=0$.

(vi) The topological closures $\overline{G \cdot x} \subset F$ of the $G$-orbits are rational varieties.

If the original tangent vector field $v$ vanishes at some point in $X$, the above conjugation holds with $\tilde{v}^{\prime}=0$.

If $X$ is a projective manifold then one may set $X_{\epsilon}=X$, and $(\tilde{X}, \tilde{v})$ is biholomorphic to $\left(\tilde{X}, \tilde{v}^{\prime}+\tilde{w}\right)$.

Proof. Let $\mathfrak{a}_{X}$ be a central Abelian subalgebra of $\mathfrak{h}_{X}$ such that $\mathfrak{h}_{X}=\mathfrak{h}_{X}^{1} \oplus \mathfrak{a}_{X}$. By Proposition 7.3, $X$ admits a small $b$-deformation $X_{\epsilon}$ and there is a finite Abelian covering $\tilde{X}_{\epsilon} \rightarrow X_{\epsilon}$ such that $\tilde{X}_{\epsilon}$ is the suspension $F \times{ }_{\Lambda} \mathbb{C}^{s}$, over a torus $T=\mathbb{C}^{s} / \Lambda$ and with fiber a Kähler manifold $F$ without non-singular vector fields, associated to a representation $\rho: \Lambda=\pi_{1}(T) \cong \mathbb{Z}^{2 s} \rightarrow \operatorname{Aut}_{\mathbb{C}}^{0}(F)$. As above $\tilde{X}_{\epsilon}$ can be seen as a $b$-deformation of a 
finite covering $\tilde{X}$ of $X$. We set

$$
\Lambda=\pi_{1}(T)=\left\langle\gamma_{1}, \ldots, \gamma_{2 s}\right\rangle .
$$

By Proposition 7.3 and Theorem 1.2, $\mathfrak{h}_{F}=\mathfrak{h}_{F}^{1}$ and $\operatorname{Aut}_{\mathbb{C}}^{0}(F)$ is a linear algebraic group. Hence the Zariski closure of $\rho(\Lambda)$ is an Abelian linear algebraic subgroup. In particular it has a finite number of connected components. By replacing $T$ by a suitable Abelian finite covering of it, we can assume that $\gamma_{i}=\exp _{1}\left(\xi_{i}\right)$ with $\xi_{i}$ holomorphic vector fields on $F$ generating an Abelian subalgebra of $\mathfrak{h}_{F}=\mathfrak{h}_{F}^{1}$.

Let us write $v=w+v^{\prime}$, where $w \in \mathfrak{h}_{X}^{1}$ and $v^{\prime} \in \mathfrak{a}_{X}$. If $v$ has zeros then $v^{\prime}=0$. The algebra $\mathfrak{a}_{X}$ is central, so $\left[v^{\prime}, w\right]=0$. Using Proposition 5.9, we see that $\mathfrak{h}_{X_{\epsilon}}^{1}=\mathfrak{h}_{X}^{1}$, $\mathfrak{a}_{X_{\epsilon}}=\mathfrak{a}_{X}$ and $\mathfrak{h}_{X_{\epsilon}}=\mathfrak{h}_{X_{\epsilon}}^{1} \oplus \mathfrak{a}_{X_{\epsilon}}$. Hence $v, w, v^{\prime}$ are holomorphic vector fields on $X_{\epsilon}$ and their lifts $\tilde{v}, \tilde{w}$ and $\tilde{v}^{\prime}$ to $\tilde{X}_{\epsilon}$ still satisfy $\tilde{v}=\tilde{w}+\tilde{v}^{\prime} \in \mathfrak{h}_{\tilde{X}_{\epsilon}}$ and $\left[\tilde{v}^{\prime}, \tilde{w}\right]=0$. There is a Lie algebra decomposition $\mathfrak{h}_{\tilde{X}_{\epsilon}}=\mathfrak{h}_{\tilde{X}_{\epsilon}}^{1} \oplus \mathfrak{a}_{\tilde{X}_{\epsilon}}$ where the elements of $\mathfrak{a}_{\tilde{X}_{\epsilon}}$ are projections on $\tilde{X}_{\epsilon}=F \times_{\Lambda} \mathbb{C}^{s}$ of linear vector fields on $\mathbb{C}^{s}$ and $\mathfrak{h}_{\tilde{X}_{\epsilon}}^{1}$ is naturally identified to a subalgebra of $\mathfrak{h}_{F}=\mathfrak{h}_{F}^{1}$. On the one hand, we have $\tilde{v}^{\prime} \in \mathfrak{a}_{\tilde{X}_{\epsilon}}$ by Remark 6.7. On the other hand, $\tilde{w}$ is an element of $h_{\tilde{X}_{\epsilon}}^{1}$ since it is a vector field with zeros. In particular we can think of $\tilde{w}$ as a vector field on $F$ which is invariant by the monodromy, that is, $\tilde{w}=\left(\rho_{\gamma}\right)_{*} \tilde{w}$ for each $\gamma \in \Lambda$ or, what is equivalent,

$$
\exp _{k}\left(\xi_{i}\right)_{*} \tilde{w}=\tilde{w} \quad \text { for } 1=1, \ldots, 2 s \text { and } k \in \mathbb{Z} .
$$

Let $H$ be the Abelian subgroup of $\operatorname{Aut}_{\mathbb{C}}^{0}(F)$ generated by the elements $\exp _{k}\left(\xi_{i}\right)$ and $\exp _{t}(\tilde{w})$, where $i=1, \ldots, 2 s, k \in \mathbb{Z}$ and $t \in \mathbb{C}$. The Zariski closure $G$ of $H$ is an Abelian linear algebraic subgroup and by replacing again $T$ by a suitable Abelian finite covering we can assume that $G$ is connected. Therefore, the group $G$ is of the form $\mathbb{C}^{p} \times\left(\mathbb{C}^{*}\right)^{q}$.

The group $\operatorname{Aut}_{\mathbb{C}}^{0}(F)$ not only acts compactifiably on $F$ in the sense of Lieberman but, by [16, Remark 2.3], it also admits a compactification $\operatorname{Aut}_{\mathbb{C}}^{0}(F)^{*}$ which is a projective variety. The group $G$ acts on $F$ as a linear algebraic subgroup of $\operatorname{Aut}_{\mathbb{C}}^{0}(F)$. The Zariski closure $G^{*}$ of $G$ in $\operatorname{Aut}_{\mathbb{C}}^{0}(F)^{*}$ compactifies the action of $G$ in $F$.

Let $x$ be a point in $F$ and denote by $G \cdot x$ its $G$-orbit. Then $G \cdot x=G / G_{x}$, where $G_{x}$ is the stabilizer of $x$. The Zariski closure $G_{x}^{*}$ of $G_{x}$ in $G^{*}$ is algebraic by GAGA. Therefore $G_{x}=G \cap G_{x}^{*}$ is an algebraic subgroup of $G$. Let $G_{x}^{0}$ be the connected component of the identity in $G_{x}$. It is a connected Abelian linear algebraic subgroup of $G$, so the quotient $G / G_{x}^{0}$ is also an Abelian linear algebraic connected group (cf. [22]). It follows that $G / G_{x}^{0}$ is isomorphic to a group of type $\mathbb{C}^{p} \times\left(\mathbb{C}^{*}\right)^{q}$. Since the orbit $G \cdot x=G / G_{x}$ is a finite quotient of $G / G_{x}^{0}$, it is again of type $\mathbb{C}^{p} \times\left(\mathbb{C}^{*}\right)^{q}$. As the action of $G$ in $F$ is compactifiable, by [28, Proposition 3.7] the orbit $G \cdot x$ is a dense Zariski open set of its closure $\overline{G \cdot x}$, which is therefore a rational variety. The fiber $F$ is covered by such orbits, so it is uniruled.

If $X$ is a projective manifold then there is no need of considering tangential deformations by virtue of Theorem 3.2. 
Remark 9.3. In the case when the tangent field $v$ vanishes at some point, the torus $T$ may be trivial, and our theorem says that the closures of the orbits of $\tilde{v}$ are rational varieties. Applied to particular vector fields, this is already the central argument in the proof of Fujiki's Theorem 1.8, and Lieberman's version in [28].

The preceding results mean that, if one forgets linear vector fields on tori, the dynamics of a holomorphic vector field on a compact Kähler manifold reduces to the dynamics of an Abelian connected linear algebraic group (thus of type $\mathbb{C}^{p} \times\left(\mathbb{C}^{*}\right)^{q}$ ) acting on a rational variety.

Acknowledgments. We are very indebted to M. Brunella for inspiring discussions and to F. Touzet who communicated to us the proof of Proposition 1.6.

This work was partially supported by the Ministerio de Educación y Ciencia of Spain, grants MTM2008-02294 and MTM2009-14163-C02-02, and by the Generalitat de Catalunya grants 2009SGR 1207 and 2009SGR 1284. The second author was supported by the Programa de Movilidad de Recursos Humanos del Plan Nacional de I-D+I 2008/2011.

\section{References}

[1] Beauville, A.: Complex Algebraic Surfaces. 2nd ed., London Math. Soc. Student Texts 34, Cambridge Univ. Press (1996) Zbl 0849.14014 MR 1406314

[2] Beauville, A.: Variétés Kähleriennes dont la première classe de Chern est nulle. J. Differential Geom. 18, 755-782 (1983) Zbl 0537.53056 MR 0730926

[3] Birkenhake, C., Lange, H.: Complex Abelian Tori. Progr. Math. 177, Birkhäuser (1999)

[4] Birkenhake, C., Lange, H.: Complex Abelian Varieties. 2nd ed., Grundlehren Math. Wiss. 302, Springer, Berlin (2004) Zbl 1056.14063 MR 2062673

[5] Blanchard, M. A.: Sur les variétés analytiques complexes. Ann. Sci. École Norm. Sup. (3) 73, 157-202 (1956) Zbl 0073.37503 MR 0087184

[6] Bogomolov, F. A.: Kähler manifolds with trivial canonical class. Izv. Akad. Nauk SSSR Ser. Mat. 38, 11-21 (1974) (in Russian) Zbl 0292.32020 MR 0338459

[7] Bosio, F.: Actions holomorphes et localement libres de groupes de Lie abéliens. Thèse de Doctorat, École Normale Supérieure de Lyon (1996)

[8] Calabi, E.: On Kähler manifolds with vanishing canonical class. In: Algebraic Geometry and Topology. A Symposium in Honor of S. Lefschetz, Princeton Univ. Press, Princeton, NJ, 7889 (1957) Zbl 0080.15002 MR 0085583

[9] Campana, F.: The class $\mathcal{C}$ is not stable by small deformations. Math. Ann. 290, 19-30 (1991) Zbl 0722.32014 MR 1107661

[10] Carrell, J. B.: Holomorphically injective complex toral actions. In: Proc. 2nd Conf. Compact Transformation Groups (Amherst, MA, 1971), Vol. 2, Lecture Notes in Math. 299, Springer, 205-236 (1972) Zbl 0252.57017 MR 0590935

[11] Carrell, J. B., Lieberman, D. I.: Holomorphic vector fields and Kähler manifolds. Invent. Math. 21, 303-309 (1973) Zbl 0253.32017 MR 0326010

[12] Carrell, J. B., Sommese, A. J.: $C^{*}$-actions. Math. Scand. 43, 49-59 (1978/79) Zbl 0416.32022 MR 0523824

[13] Cathelineau, J.-L.: Déformations équivariantes d'espaces analytiques complexes compacts. Ann. Sci. École Norm. Sup. (4) 11, 391-406 (1978) Zbl 0401.32010 MR 0521637 
[14] Demailly, J.-P., Paun, M.: Numerical characterization of the Kähler cone of a compact Kähler manifold. Ann. of Math. (2) 159, 1247-1274 (2004) Zbl 1064.32019 MR 2113021

[15] Fujiki, A.: Closedness of the Douady spaces of compact Kähler spaces. Publ. Res. Inst. Math. Sci. 14, 1-52 (1978) Zbl 0409.32016 MR 0486648

[16] Fujiki, A.: On automorphism groups of compact Kähler manifolds. Invent. Math. 44, 225-258 (1978) Zbl 0367.3200 MR 0481142

[17] Fujiki, A.: On the structure of compact complex manifolds in $\mathcal{C}$. In: Algebraic Varieties and Analytic Varieties (Tokyo, 1981), Adv. Stud. Pure Math. 1, North-Holland, Amsterdam, 231302 (1983) Zbl 0513.32027 MR 0715653

[18] Girbau, J., Nicolau, M.: On deformations of holomorphic foliations. Ann. Inst. Fourier (Grenoble) 39, 417-449 (1989) Zbl 0659.32019 MR 1017285

[19] Gómez-Mont, X.: Transverse deformations of holomorphic foliations. In: The Lefschetz Centennial Conference, Part I (Mexico City, 1984), Contemp. Math. 58, Amer. Math. Soc., Providence, RI, 127-139 (1986) Zbl 0607.32014 MR 0860409

[20] Hall, R.: On algebraic varieties which possess finite continuous commutative groups of birational self-transformations. J. London Math. Soc. 30, 507-511 (1955) Zbl 0065.14001 MR 0073276

[21] Holmann, H.: On the stability of holomorphic foliations with all leaves compact. In: Variétés analytiques compactes (Nice, 1977), Lecture Notes in Math. 683, Springer, Berlin, 217-248 (1978) Zbl 0403.32015 MR 0517527

[22] Humphreys, J. E.: Linear Algebraic Groups. Grad. Texts in Math. 21, Springer, New York (1975) Zbl 0325.20039 MR 0396773

[23] Kodaira, K., Spencer, D. C.: On deformations of complex analytic structures. I, II. Ann. of Math. (2) 67, 328-466 (1958) Zbl 0128.16901 MR 0112154

[24] Kollár, J.: Shafarevich Maps and Automorphic Forms. M. B. Porter Lectures. Princeton Univ. Press, Princeton, NJ (1995) Zbl 0871.14015 MR 1341589

[25] LeBrun, C., Poon, Y. S.: Twistors, Kähler manifolds, and bimeromorphic geometry. II. J. Amer. Math. Soc. 5, 317-325 (1992) Zbl 0766.53051 MR 1137099

[26] Lichnerowicz, A.: Variétés kähleriennes et première classe de Chern. J. Differential Geom. 1, 195-223 (1967) Zbl 0167.20004 MR 0226561

[27] Lieberman, D. I.: Holomorphic vector fields on projective varieties. In: Several Complex Variables (Williamstown, MA, 1975), Proc. Sympos. Pure Math. 30, Amer. Math. Soc., 273-276 (1977) Zbl 0365.32021 MR 0454086

[28] Lieberman, D. I.: Compactness of the Chow scheme: applications to automorphisms and deformations of Kähler manifolds. In: Fonctions de plusieurs variables complexes, III (Sém. François Norguet, 1975-1977), Lecture Notes in Math. 670, Springer, Berlin, 140-186 (1978) Zbl 0391.32018 MR 0521918

[29] Manjarín, M.: Normal almost contact structures and non-Kähler compact complex manifolds. Indiana Univ. Math. J. 57, 481-507 (2008) Zbl 1142.32009 MR 2400265

[30] Matsushima, Y.: Holomorphic vector fields and the first Chern class of a Hodge manifold. J. Differential Geom. 3, 477-480 (1969) Zbl 0201.25902 MR 0273553

[31] Severi, F.: Funzione quasi abeliane. Pontificiae Academiae Scientiarum Scripta Varia, Vol. 4, Città del Vaticano (1947)

[32] Ueno, K.: Classification Theory of Algebraic Varieties and Compact Complex Spaces. Lecture Notes in Math. 439, Springer, Berlin (1975) Zbl 0299.14007 MR 0506253

[33] Wang, H.-C.: Complex parallisable manifolds. Proc. Amer. Math. Soc. 5, 771-776 (1954) Zbl 0056.15403 MR 0074064 